\title{
Das Schwimmen der Talitridae \\ (Crustacea, Amphipoda): \\ Funktionsmorphologie, Phänomenologie und Energetik
}

\author{
Friedhelm Vogel \\ Zoologisches Institut der Universität Münster, \\ Abteilung Physiologie und Ókologie; \\ Badestraße 9, D-4400 Münster, Bundesrepublik Deutschland
}

\begin{abstract}
The swimming of the Talitridae (Crustacea, Amphipoda): Functional morphology, phenomenology, and energetics. The Talitridae, well-known for their jumping behaviour, swim with help of the tail-flip. This movement of the abdomen is also known from other amphipods like the Gammaridae which are normally not able to move by jerks outside the water. The suspected homology between the tail-flip when swimming and the jerky movement of the abdomen when jumping gave rise to this investigation, mainly based on high frequency film recordings, on the swimming of Hyale nilssonii, Orchestia cavimana, and Talitrus saltator (family Talitridae) as well as three related species of the families Gammaridae and Corophiidae. Comparative morphometrical and SEM-studies on the habitus of the species and the build of the involved limbs reveal the rather uniform construction of the Gammaridea; functional adaptation to the environment and to the way of living become apparent in minor alterations. The joints of the pleopods and uropods show a clear structural adaptation to the mechanical strain during swimming. The pleopods are moved metachronally in all examined species; angular velocity and rate of beating indicate the efficiency of the swimming movement. In the Talitridae, the metachronal beat of the pleopods is nearly always coupled with the tail-flip while in the Gammaridae and Corophiidae the tail-flip, in addition to the beat of the pleopods, is mostly used for a start from the subsoil or for a change in swimming direction. H. nilssonii, Gammarus locusta, and Corophium volutator, all inhabitants of the tidal zone in the North Sea shallows, turned out to be the "best" swimmers while the (semi-) terrestrially living species, $O$. cavimana and $T$. saltator, proved to be rather "poor" swimmers. This clearly indicates the ecological significance of swimming for the different species. Furthermore, the tailflip is found to be of rather subordinate importance. It contributes to a higher velocity if used moderately but is rather obstructive if a large angle is covered while extending and flexing the abdomen. The efficiency of swimming is inversely proportional to the efficiency of jumping in the three talitridean species. Thus, better adaptation to terrestrial life is accompanied by loss of swimming efficiency. Examined under the aspect of locomotional homology, it is concluded that the tail-flip used while swimming is homologous to the jerky movement of the abdomen used for jumping. The comparison of the swimming performance of the examined species with other crustaceans and some fishes illustrates the over-all good results of the Gammaridea.
\end{abstract}

\section{EINLEITUNG}

Die Amphipoda bilden eine weitverbreitete und ökologisch sehr vielseitige Gruppe der Malacostraca. Sie kommen in Meerestiefen von $10000 \mathrm{~m}$ (Altevogt, 1971), in 
Quellen unter Tage und sogar "... high in mountains..." (Abele, 1982) vor. Echte terrestrische Arten finden sich nur in der Familie der Talitridae (Hurley, 1968), die zur größten Unterordnung, Gammaridea, gehört. Aber auch diese terrestrischen "Flohkrebse", die im Deutschen durch ihre springende und hüpfende Bewegung namengebend für die ganze Ordnung waren, haben die Fähigkeit zu schwimmen noch nicht verloren, auch wenn sie starke Adaptationen an die terrestrische Lebensweise zeigen wie z. B. gedrungeneren Körperbau, allmähliche Reduktion der Pleopoden oder Schwimmbeine (Hurley, 1968) und Verkürzung - verbunden mit kompakterem und stabilerem Bau - der zum Springen wichtigen Uropoden oder Stützbeine.

Untersuchungen an Amphipoden beschränkten sich bisher zumeist auf die Gebiete der Taxonomie, Zoogeographie, Populationsbiologie und Aktivitätsrhythmik. Die funktionsmorphologischen Anpassungen an die Besonderheiten des jeweiligen Lebensraumes fanden wenig Beachtung. Pardi \& Papi (1961, zusammenfassende Darstellung; weitere Literatur siehe Arendse, 1980) befaßten sich eingehend mit der Orientierung der Talitridae an Land. Auch Bracht (1978, unveröffentlicht; 1980a, 1980b) wähite als Untersuchungsobjekte Vertreter der Talitridae; er analysierte das Sprungvermögen dieser Tiere mit Hilfe der Hochgeschwindigkeitskinematographie und der Stroboskopmethode.

Beobachtungen zum Schwimmen von Amphipoden führten meist nur zu recht allgemeinen Bemerkungen über Schwimmlage und Güte des Schwimmvermögens sowie über Einsatz des Abdomenschlages beim Schwimmen. Allgemein gelten die Talitridae, verglichen mit anderen Amphipoden wie z. B. Gammarus, als schlechte Schwimmer (Gerstaecker \& Ortmann, 1901; Smallwood, 1903, 1905; Mortensen, 1921; Schlienz, 1922; Dudich, 1927; Dahl, 1946; Bowers, 1964). Talitrus eastwoodae kann sich unter Wasser nicht einmal mehr vom Untergrund abheben (Lawrence, 1953), und Talitrus saltator soll sich nur durch gemeinsame Anstrengung der Pleopoden und des ganzen Abdomens gerade noch ". . . schwebend erhalten . .." können (Schellenberg, 1929, 1942); dagegen gibt Verwey (1927) für T. saltator (Körperlänge ca. $10 \mathrm{~mm}$ ) eine Schwimmgeschwindigkeit von $5-6 \mathrm{~cm} \cdot \mathrm{s}^{-1}$ an. Dieses ist die einzige konkrete Zahlenangabe zur Schwimmgeschwindigkeit in der Amphipodenliteratur. Spelaeorchestia koloana kann zwar so gut wie andere Talitridae schwimmen, zeigt aber " . . considerable difficulty in breaking the surface tension to return to the land ..." (Bousfield \& Howarth, 1976).

Schon diese wenigen, recht unpräzisen Außerungen deuten die Komplexität des Schwimmvorganges bei den Amphipoda und insbesondere bei den Talitridae an. Hinzu kommt die vermutete funktionsmorphologische Homologie des Sprungvorganges der Talitridae mit dem Abdomenschlag während des Schwimmens (Bracht, 1980b), was durch Befunde zum funktionellen Bau der Abdomenmuskulatur von Orchestia cavimana (Vogel, 1986) gestützt wird. Um hierüber nähere Aufschlüsse sowohl qualitativer als auch quantitativer Art zu erlangen, setze ich erstmalig die Hochfrequenzkinematographie zur Untersuchung schwimmender Amphipoda ein. Rasterelektronenmikroskopische Untersuchungen zur Pleopoden- und Uropodenmorphologie ergänzen die Befunde. 


\section{VERSUCHSTIERE UND ANGEWANDTE VERFAHREN}

\section{Untersuchte Arten, deren Lebensräume und Lebensweise}

Als Untersuchungsobjekte wählte ich drei Arten der Familie Talitridae: Orchestia cavimana Heller, 1865, Talitrus saltator (Montagu, 1808) und Hyale nilssonii (Rathke, 1843). Vergleichsdaten gewann ich an Gammarus locusta (Linné, 1758) und G. pulex (Linné, 1758), Familie Gammaridae, sowie an Corophium volutator (Pallas, 1766), Familie Corophiidae.

O. cavimana sammelte ich in den Geröll- und Steinpackungen des Dortmund-EmsKanals bei Münster-Hiltrup oberhalb der Wasserlinie. Diese Tiere ließen sich gut in Zucht halten und vermehren, wie dieses von Lehmann (1971, unveröffentlicht) und Bracht (1978, unveröffentlicht; 1980b) beschrieben wird. $O$. cavimana stand mir so auch im Winter in ausreichender Zahl zur Verfügung und bot sich dadurch für Vorversuche jeglicher Art an.

T. saltator lebt am oberen Spülsaumrand von Sandstränden unter angeschwemmtem Pflanzenmaterial und Strandgut. Zum Schwimmen hat diese Art also normalerweise keine Gelegenheit mehr. Wie $O$. cavimana ist $T$. saltator hauptsächlich nachtaktiv. Meine Versuchstiere stammen vom Oststrand von Norderney, wo ich sie unter angeschwemmten Holzplanken fand.

Als dritte Art der Talitridae untersuchte ich $H$. nilssonii. Diese Flohkrebse gehen nicht an Land, sondern halten sich im Pflanzenbewuchs innerhalb der Gezeitenzone auf. Ich fand $H$. nilssonii im Fucus-vesiculosus-Gürtel auf den bei Flut regelmäßig überfluteten und dem Küstenschutz dienenden Steinwällen im Wattenmeer vor der Biologischen Station Carolinensiel. $H$. nilssonii wie auch $T$. saltator wurden möglichst bald (innerhalb von 24 Stunden nach dem Sammeln) gefilmt, da diese Krebse in Zuchtbecken nur wenige Tage überlebten.

Die drei beschriebenen Arten der Talitridae repräsentieren drei Stufen auf dem Weg zur terrestrischen Lebensweise. $H$. nilssonii ist als litorale Art noch sehr stark an das Wasser gebunden. $O$. cavimana lebt zwar oberhalb der Wasserlinie, ist aber noch nicht so gut an das terrestrische Leben angepaßt wie $T$. saltator; für letzteren ist es ausreichend, wenn der Sand noch geringfügig feucht ist. T. saltator zeigt auch entsprechend das beste Sprungvermögen dieser drei Arten (Bracht, 1980b).

Mit $H$. nilssonii vergesellschaftet findet man G. locusta. Er ist ebenfalls ein Bewohner des Pflanzenbewuchses im Litoral der Wattenmeere und eignet sich daher gut als Vergleichsart zu den genannten Talitridae. Als Süßwasser-Vertreter der Gammaridae wählte ich G. pulex. Er bewohnt die pflanzenbewachsenen Stellen fließender Gewässer. Dort tritt er in klaren, sauberen Bächen in großer Zahl auf. Meine Versuchstiere stammen größtenteils aus dem Hangsbach bei Hangsbeck im westlichen Münsterland. Die Gammaridae wurden ebenfalls möglichst bald nach dem Sammeln gefilmt.

Die Vertreter der Gattung Corophium bieten aufgrund ihres Habitus andere Voraussetzungen für das Schwimmen und insbesondere für den Abdomenschlag als die Talitridae und die Gammaridae. Daher schloß ich $C$. volutator in meine Untersuchungen ein. Dieser Krebs lebt in großer Zahl im Schlick des Wattenmeeres, wo er U-förmige Röhren anlegt. Vom Eingang der Röhre aus wird der Schlick unter Mithilfe der langen zweiten Antennen "abgeweidet". Während der ablaufenden bzw. auflaufenden Flut sieht man viele dieser Wattkrebse in den kleinen Prielen hin- und herschwimmen. 
Meine Versuchstiere stammen wiederum aus dem Wattenmeer vor der Biologischen Station Carolinensiel. Sie konnten über Wochen in einem zur Hälfte mit Schlick, zur anderen Hälfte mit Meerwasser gefüllten, gut belüfteten Aquarium gehalten werden (siehe auch Meadows \& Reid, 1966).

\section{Methoden}

Für die vergleichend-phänomenologischen und energetischen Untersuchungen des Schwimmens der erwähnten Arten wurde die Hochfrequenzkinematographie eingesetzt. Zur Verfügung stand eine 16-mm-Hochfrequenzkamera vom Typ LOCAM (Modell 51-0002, Redlake Corporation, Campbell, Calif., USA) mit einem 75-mm-Objektiv (Kerm Switar $1: 1$,9) und Zwischenringen von 15 bis $30 \mathrm{~mm}$ Länge. Die Aufnahmegeschwindigkeit betrug $500 \mathrm{~B} / \mathrm{s}$, die Belichtungszeit pro Bild 1/1500 s; nach der Beschleunigungsphase der Kamera wurde die eingestellte Bildfrequenz innerhalb eines Spielraumes von $\pm 2 \mathrm{~B} / \mathrm{s}$ gehalten. Die Beleuchtungseinheit bestand aus zwölf Punktstrahlern von jeweils $150 \mathrm{~W}$ (zusammen $1800 \mathrm{~W}$ ). Dadurch konnte eine Blende von 11 bis 16 erreicht werden. In Abständen von $10 \mathrm{~ms}$ wurde der Filmrand mit Zeitmarken versehen, die die genaue Errechnung der Filmgeschwindigkeit erlaubten. Für erste Übersichtsaufnahmen stellte ich eine Geschwindigkeit von $300 \mathrm{~B} / \mathrm{s}$ bei $5 \mathrm{~mm}$ Zwischenring und $900 \mathrm{~W}$ Beleuchtung ein, wodurch immerhin noch Blende 4 erreicht wurde. Als Filmmaterial verwendete ich handelsübliches Negativ- und Positivmaterial (Gevapan $30 \mathrm{~N}$ mit 19 DIN, $36 \mathrm{~N}$ mit 24 DIN und $30 \mathrm{R}$ mit 17 DIN). Durch die Wahl der Optik konnten die Versuchstiere auf dem Film mit einem Verkleinerungsfaktor von 0,28 bis 0,42 abgebildet werden.

Die Versuchstiere befanden sich während der Aufnahme in einer Glasküvette mit den Innenmaßen $58 \mathrm{~mm} \times 80 \mathrm{~mm} \times 11 \mathrm{~mm}(\mathrm{~B} \times \mathrm{H} \times \mathrm{T})$. Die Frontscheibe, durch die gefilmt wurde, bestand aus $1 \mathrm{~mm}$ dickem Glas, die Rückwand wurde für Übersichtsaufnahmen mit schwarzem Schaumstoff versehen. Für quantitative Messungen schob ich einen weißen Raster auf schwarzem Grund (Maschenweite $2 \mathrm{~mm}$ ) ein; außerdem wählte ich den Bildausschnitt so, daß nur wirklich freies, nicht durch Scheiben behindertes Schwimmen gefilmt wurde.

Umgeben war die Glasküvette von einer größeren Kühlküvette (Innenmaße: $145 \mathrm{~mm} \times 80 \mathrm{~mm} \times 51 \mathrm{~mm}$ ), die mit einem Gemisch aus Wasser und Eis gefüllt wurde. So konnte die Wassertemperatur in der Versuchsküvette in einem annehmbaren Bereich gehalten werden. Zu Beginn betrug sie (je nach Dauer der Einstellarbeiten) 20 bis $25^{\circ} \mathrm{C}$, sie stieg dann während der Aufnahmen auf maximal $28^{\circ} \mathrm{C}$ an. Lehmann (1971; unveröf.) gibt als Vorzugstemperatur 15- bis $17-\mathrm{mm}$ großer Exemplare von $O$. cavimana im August 22 bis $27^{\circ} \mathrm{C}$ an (meine Filmaufnahmen erfolgten während der Sommermonate von Ende Mai bis Mitte September 1981 und 1982), als Letaltemperatur 35 bis $40^{\circ} \mathrm{C}$, und Schellenberg (1942) fand G. pulex an einer Stelle, wo die Jahresdurchschnittstemperatur immerhin $19,7^{\circ} \mathrm{C}$ betrug.

Vor der Aufnahme wurden die Versuchstiere mindestens fünf Stunden bei Raumtemperatur (ca. $24^{\circ} \mathrm{C}$ ) gehalten. Für Talitrus saltator, Hyale nilssonii, Gammarus locusta und Corophium volutator fültte ich die Versuchsküvette mit Meerwasser vom jeweiligen Fundort (die Salinität betrug 28 bis $32 \%$ ), für Gammarus pulex entsprechend mit Süßwasser vom Fundort und für Orchestia cavimana mit Leitungswasser, woran diese 
Tiere durch die Zucht gewöhnt waren. Gleich nach einer Aufnahme wurden die Versuchstiere auf einer Analysenwaage (Sartorius Selecta-Standard, $200 \mathrm{~g} / 0,1 \mathrm{mg}$ ) nach vorsichtigem Abtupfen noch anhaftenden Wassers gewogen und anschließend in 70 prozentigem Alkohol konserviert.

Die quantitative Auswertung der Filme erfolgte Bild für Bild mit Hilfe eines Analysenprojektors vom Typ Lafayette Analyzer AAP 300 (Lafayette, Indiana, USA). Die einzelnen Filmbilder projizierte ich über zwei Spiegel auf einen Tisch (die Spiegel nahmen jeweils einen Winkel von $45^{\circ}$ zum Lichtstrahl ein $i$ der Vergrößerungsmaßstab konnte über Anderung der Entfernung des Projektors vom ersten Spiegel variiert werden) und zeichnete sie auf DIN-A4-Papier um, das mit dem obenerwähnten Raster jedoch exakt 1:10 vergrößert - bedruckt war.

Da neben der Körpergeschwindigkeit auch verschiedene Winkelgeschwindigkeiten ermittelt werden mußten, konnten für die quantitative Auswertung nur Filmaufnahmen berücksichtigt werden, die bestimmte Voraussetzungen erfüllten:

- Die Krebse sollten frei und unbehindert schwimmen; es sollte sich weder um Beginn noch Ende der Bewegung handeln. Dieses wurde durch die Wahl des Bildausschnittes, der die Versuchsküvettenränder nicht mit einbezog, an denen sich die Tiere am liebsten aufhielten, weitestgehend vorgegeben.

- Die Krebse sollten parallel zur Filmebene schwimmen. Dazu trug die geringe Tiefe der Versuchsküvette bei.

- Die Krebse sollten, von der Kamera aus gesehen, genau in Seitenansicht schwimmen, da nur so die Winkelgeschwindigkeiten von Pleopoden und Abdomen exakt meBbar sind.

Aufgrund dieser Bedingungen konnte ich nur einen Teil der filmisch erfaßten Schwimmzyklen (ein Schwimmzyklus umfaßt einen kompletten Abdomenschlag und/ oder eine vollständige Sequenz von Pleopodenschlägen) bezüglich aller gewünschten Meßwerte analysieren; Körpergeschwindigkeit und Schwimmzykluslänge ließen sich darüber hinaus noch bei einer Reihe von Übersichtsaufnahmen bestimmen (Tab. 1).

Tab. 1. Anzahl der filmisch dokumentierten, unbehinderten Schwimmzyklen

\begin{tabular}{|lccccc|}
\hline Art & $\begin{array}{c}\text { Schwimm- } \\
\text { zyklen } \\
\text { (Gesamt- } \\
\text { zahl) }\end{array}$ & $\begin{array}{c}\text { (a) Uber- } \\
\text { sichts- } \\
\text { aufnahmen }\end{array}$ & $\begin{array}{c}\text { davon } \\
\text { quantitativ } \\
\text { auswertbar }\end{array}$ & $\begin{array}{c}\text { (b) Auf- } \\
\text { nahmen zur } \\
\text { quantitativen } \\
\text { Auswertung }\end{array}$ & $\begin{array}{c}\text { davon } \\
\text { auswertbar }\end{array}$ \\
\hline Orchestia cavimanativ & 244 & 208 & 40 & 36 & 7 \\
Talitrus saltator & 54 & 0 & 0 & 54 & 3 \\
Hyale nilssonii & 212 & 134 & 17 & 78 & 11 \\
Gammarus locusta & 154 & 38 & 7 & 116 & 17 \\
Gammarus pulex & 129 & 0 & 0 & 129 & 17 \\
Corophium volutator & 293 & 174 & 0 & 119 & 16 \\
\hline
\end{tabular}

Durch die Filmgeschwindigkeit von $500 \mathrm{~B} / \mathrm{s}$ wurde ein Schwimmzyklus je nach Dauer in 20 bis 45 Einzelbilder zerlegt. Die Einzelbilder zeichnete ich, wie schon oben beschrieben, um; dabei wurden die Versuchstiere genau 10 fach vergrößert abgebildet. In die Zeichnungen trug ich jeweils vier Meßpunkte (für Corophium drei) ein (Abb. 1): 
$\mathrm{P}=$ Mittelpunkt des vierten Pereionsegmentes, zugleich ungefährer Schwerpunkt des Körpers ohne Abdomen (ein genauer Schwerpunkt läßt sich nicht angeben, da er bei Krümmung und Streckung des Körpers verlagert wird);

$\mathrm{A}=$ Drehpunkt zwischen siebtem Pereionsegment und erstem Abdominalsegment;

$\mathrm{T}=$ Telsonspitze (dieser Meßpunkt entfiel bei Corophium, da er fast deckungsgleich mit U war);

$\mathrm{U}=$ Spitze der ersten Uropoden bei den Talitridae und den Corophiidae bzw. der dritten Uropoden bei den Gammaridae.

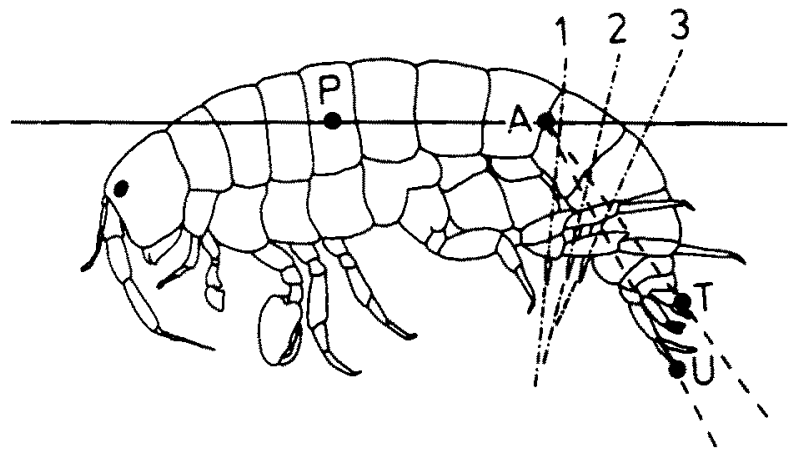

Abb. 1. Meßpunkte und -achsen zur quantitativen Filmanalyse. $P=$ Mittelpunkt des vierten Pereionsegmentes; $\mathrm{A}=$ Drehpunkt zwischen siebtem Pereionsegment und erstem Abdominalsegment; $\mathrm{T}=$ Telsonspitze; $\mathrm{U}=$ Uropodenspitze; $1-3=$ Pleopodenlängsachsen; $\overline{\mathrm{PA}}=$ Körperlängsachse; $\overline{\mathrm{AT}}, \overline{\mathrm{AU}}=$ Abdomenlängsachse

Für $\mathrm{P}$ und $\mathrm{A}$ bestimmte ich die Lineargeschwindigkeiten durch Messung der jeweils nach drei Bildern zurückgelegten Wegstrecke mit Hilfe einer Handlupe mit Meßskala (Einteilung in $1 / 10-\mathrm{mm}$-Schritte; durch die 10 fache Vergrößerung der Umzeichnungen gegenüber den Originalmaßen der Versuchstiere betrug die Ablesegenauigkeit also $\pm 1 / 100 \mathrm{~mm})$. Da die Schwimmrichtungen der Versuchstiere sehr unterschiedlich waren jede Richtung zwischen $0^{\circ}$ ( = senkrecht nach unten) und $180^{\circ}$ (= senkrecht nach oben) war möglich - und die Schwerkraft einen nicht geringen Einfluß auf die Geschwindigkeit ausübt, wurden die Lineargeschwindigkeiten um die ermittelten Sinkgeschwindigkeiten (Tab. 2) nach dem Vektorsubtraktionsgesetz korrigiert. Die Sinkgeschwindigkeiten bestimmte ich anhand von Filmaufnahmen, in denen sich intakte Tiere herabsinken ließen. Bei den in Tab. 2 angegebenen Werten handelt es sich um Mittelwerte verschiedener, aber in etwa gleich großer Tiere. Je kleiner die Versuchstiere sind, desto langsamer sinken sie nach unten.

Die Stellungen des Abdomens und der drei Pleopodenpaare sind durch die Winkel der entsprechenden Achsen zur Körperlängsachse charakterisiert, wobei ich als Körperlängsachse die Gerade durch die Punkte $\mathrm{P}$ und A definiere. Die Längsachsen der drei Pleopodenpaare sind in Abb. 1 mit 1 bis 3 bezeichnet. Für das Abdomen sind die Achsen $\overline{\mathrm{AT}}$ und $\overline{\mathrm{AU}}$ maßgebend. Der Winkel von $180^{\circ}$ entspricht dabei der gestreckten Abdomenhaltung; die Beugung des Abdomens drückt sich in kleiner werdendem Winkel aus. Entsprechendes gilt für die Pleopoden; deren Winkelstellung ließ sich nur so lange 
Tab. 2. Sinkgeschwindigkeiten der untersuchten Arten, ermittelt nach Filmaufnahmen herabsinkender intakter Tiere

\begin{tabular}{|ccc|}
\hline Art & $\begin{array}{c}\text { Sinkgeschwindigkeit } \\
{\left[\mathrm{m} \cdot \mathrm{s}^{-1}\right]}\end{array}$ & $\begin{array}{c}\text { Körperlänge } \\
{[\mathrm{mm}]}\end{array}$ \\
\hline Fa milie Talitridae & & \\
Orchestia cavimana & 0,030 & 20 \\
Talitrus saltator & 0,028 & 12 \\
Hyale nilssonii & 0,026 & 10 \\
Fa milie Gammarida e & & \\
Gammarus locusta & 0,026 & 5 \\
Gammarus pulex & bis 0,028 & bis 15 \\
& 0,026 & 5 \\
Fa mili e Corop hi i da e & bis 0,028 & 15 \\
Corophium volutator & & 6 \\
\hline
\end{tabular}

verfolgen, wie sie nicht von den Epimeren und Coxalplatten verdeckt wurden. Dieses war meist bei einem Winkel kleiner als 20 bis $30^{\circ}$ der Fall.

Ein weiteres Maß für die Bewegung des Abdomens bzw. der Pleopoden ist die Winkelgeschwindigkeit. Diese ermittelte ich anhand der Verlagerung der betreffenden Achse von Bild zu Bild. Es wurde also nicht die Änderung des Winkels zur Körperlängsachse von einem Bild zum nächsten gemessen, sondern die direkte Lageänderung der Abdomen- bzw. Pleopodenlängsachse. Dadurch vermied ich die zusätzliche Ungenauigkeit, die durch die - wenn auch geringfügige - Verlagerung der Körperlängsachse bei Krümmung oder Streckung des Pereions hinzugekommen wäre. Bewegungen des Abdomens und der Pleopoden nach vorn in Richtung Cephalothorax und damit in Richtung der Fortbewegung des Tieres betrachte ich als positiv, entgegengesetzte Bewegungen als negativ.

Qualitative Aussagen gewann ich durch wiederholtes Ansehen der Filme auf einem Steenbeck-Schneidetisch ST 1201 (Steenbeck, Hamburg), an dem ich auch die Sinkgeschwindigkeiten der einzelnen Arten ermittelte.

Für die Rasterelektronenmikroskopie wurden in 70prozentigem Alkohol fixierte Exemplare in absolutem Alkohol gewaschen, zwei Tage luftgetrocknet und anschlieBend mit Gold bedampft. Als Gerät stand ein Rasterelektronenmikroskop vom Typ LeitzAMR 1000 (Leihgabe der Deutschen Forschungsgemeinschaft, Projekt Al 13/11) zur Verfügung. Die morphometrischen Messungen erfolgten unter einem Wild-Binokular M 5 (Wild, Heerbrugg, Schweiz) mit Meßokular bei 6- bis 25facher Vergrößerung.

\section{ERGEBNISSE}

Bevor ich auf die Phänomenologie und Energetik des Schwimmens eingehe, beschreibe ich im folgenden kurz die Morphologie der untersuchten Arten unter Herausarbeitung der für das Schwimmen wichtigen Besonderheiten. 


\section{Vergleichend-morphologische und morphometrische Untersuchungen}

Der in Cephalothorax, Pereion und Abdomen gegliederte Körper der meisten Amphipoda ist lateral komprimiert, wodurch es für diese Krebse schwieriger ist, sich an Land aufrecht zu halten, als etwa für die dorso-ventral abgeflachten Isopoda (Hurley, 1968). Die Coxen der Pereiopoden sind plattenartig verbreitert und bilden zusammen mit der Bauchwand ein nach unten offenes Gewölbe, das als Schutzraum für die Kiemen und bei den Weibchen für die sich entwickelnde Brut dient. Caudad setzt sich dieses Gewölbe durch seitliche Verlängerungen der Rückenschilder der ersten drei Abdominalsegmente (des Metasoms), durch die sogenannten Epimeren, fort. In diesem hinteren Schutzraum sind die Pleopoden inseriert.

Die Talitridae scheinen gedrungener und kompakter gebaut zu sein als die Gammaridae und Corophiidae ( $\mathrm{Abb}$. 2); in der Relation von Körpergewicht zu Körperlänge verhalten sich jedoch die untersuchten Arten recht ähnlich. Lediglich Talitrus saltator ist relativ etwas schwerer. Dadurch daß bei den Talitridae neben den Coxalplatten auch die Basen der fünften bis siebten Pereiopoden stark verbreitert sind, wirken diese Amphipoda bei gleicher Länge und annähernd gleichem Gewicht größer und plumper als die Gammaridae und Corophiidae.

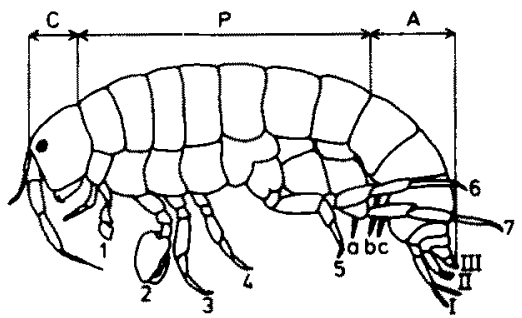

o. covimana
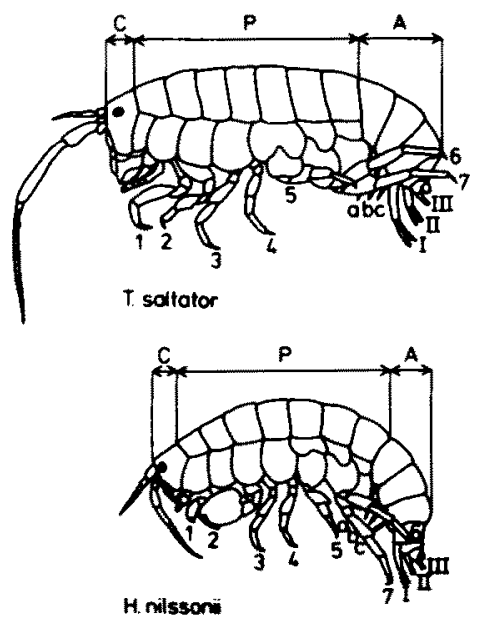

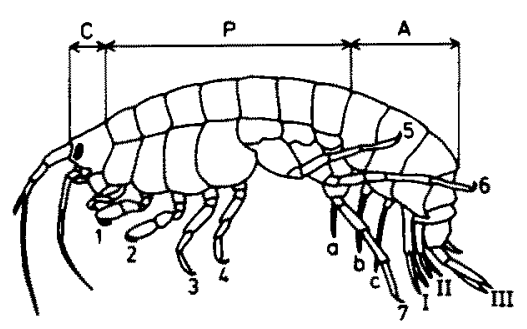

G. locusta
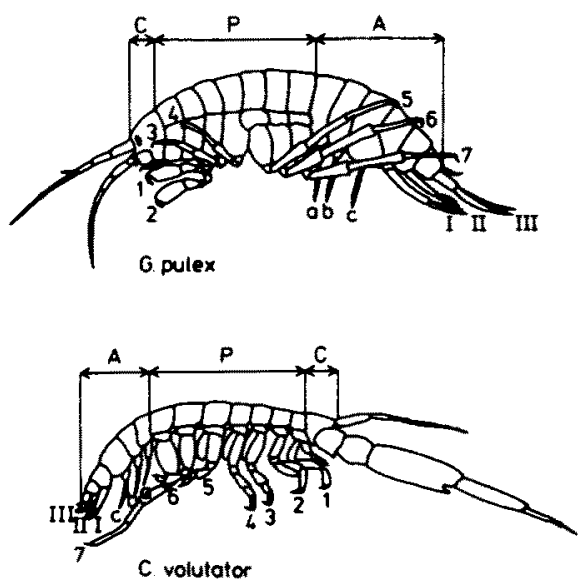

Abb. 2. Habitus der untersuchten Arten. $\mathrm{C}=$ Cephalothorax; $\mathrm{P}=$ Pereion; $\mathrm{A}=$ Abdomen; $_{1-7}=$ Pereiopoden; $\mathrm{a}-\mathrm{C}=$ Pleopoden; I-III = Uropoden (O. cavimana nach Kinzelbach, 1972; T. saltator, $H$. nilssonii, G. locusta und $C$. volutator nach Sars, 1895; G. pulex verändert nach Heinze, 1932) 


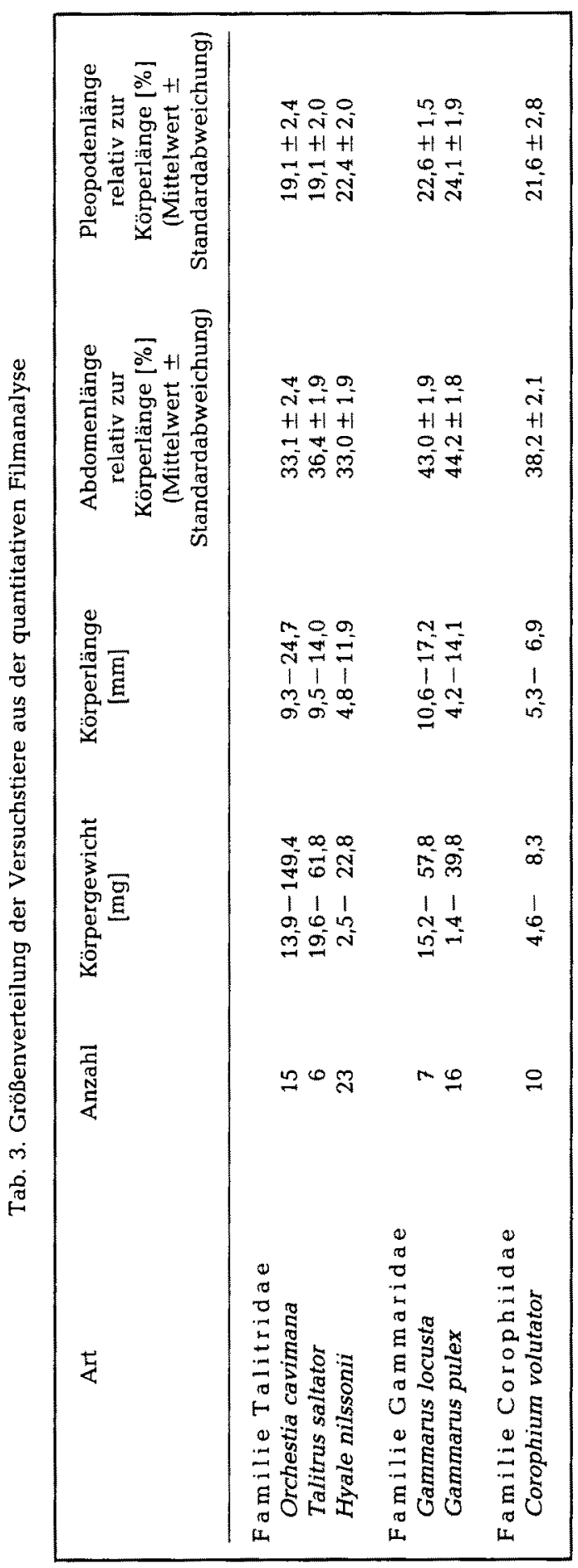


Corophium fällt dadurch auf, daß der Körper mehr oder weniger dorso-ventral abgeflacht ist, die amphipodentypische laterale Kompression also nicht mehr besteht und daß die Coxalplatten kaum verbreitert sind, wodurch die Tiere bei gleichem Gewicht auch noch kleiner als die Gammaridae wirken. Diese Krebse sind allerdings im allgemeinen recht klein, verglichen mit den Talitridae und den Gammaridae (vgl. Tab. 3).

Die ersten Antennen der Talitridae sind in der Regel viel kleiner und schwächer als die zweiten, nur bei Hyale nilssonii erreichen sie noch die halbe Länge der zweiten. Auch bei Corophium volutator sind die zweiten Antennen größer als die ersten, und sie sind auffallend kräftig gebaut; bei den Männchen können sie sogar Körperlänge und in den Basalgliedern auch fast Körperhöhe erreichen. Durch ihr Gewicht und ihre Länge bieten sie bei Beschleunigungen ein nicht zu vernachlässigendes Trägheitsmoment. Im Gegensatz dazu sind die Antennen von Gammarus nicht übermäßig dick. Die ersten Antennen sind mindestens so lang wie die zweiten, oft sogar länger als diese.

Weitere wichtige Unterschiede zwischen den Familien bestehen im Bau und in den Abmessungen des Urosoms. Bei den Talitridae sind die drei Urosomsegmente schmal und kompakt gebaut, wodurch die Beweglichkeit zwischen den Urosomsegmenten fast nicht mehr gegeben ist, ähnlich ist es bei den Corophiidae; bei den Gammaridae dagegen sind die Urosomsegmente und besonders auch die Metasomsegmente größer. Dieses drückt sich im Verhältnis der Abdomenlänge zur Gesamtkörperlänge aus. So beträgt der prozentuale Anteil des Abdomens an der Körperlänge bei den Talitridae durchschnittlich $33,5 \%(\mathrm{n}=44$; Standardabweichung $=\mathrm{SD}= \pm 2,3 \%)$, bei $C$. volutator $38,2 \%(\mathrm{n}=10 ; \mathrm{SD}= \pm 2,1 \%$ ) und bei den Gammaridae sogar $43,8 \%$ ( $\mathrm{n}=22$; $\mathrm{SD}= \pm 1,9 \%$ ) (vgl. Tab. 3). Diese Relationen bleiben auch mit zunehmender Körpergröße bestehen (Abb, 3 A). Die relative Größe des Abdomens ist sicherlich ein wichtiger Faktor, einerseits für die Effektivität des Abdomenschlags, wofür beim Rückschlag (Streckung des Abdomens) ein längeres Abdomen von Vorteil ist, beim Vorschlag (Beugung des Abdomens) jedoch ein kürzeres, andererseits aber auch für die Beweglichkeit des Abdomens, die bei kürzerem Abdomen größer ist. Im folgenden werde ich statt der deutschen Bezeichnungen "Rückschlag" und "Vorschlag" die funktionell exakteren englischen Begriffe "Powerstroke" und "Returnstroke" verwenden.

\section{Morphologie der Pleopoden}

Die Pleopoden der untersuchten Arten sind vom Grundschema her gleich gebaut. Sie bestehen jeweils aus einem ungegliederten Protopoditen, der zwei vielgliedrige, fiedrig beborstete Äste trägt, den Endo- und den Exopoditen. Der Protopodit besitzt distal auf der Innenseite einige kleine Borsten, die Schellenberg (1942) als "Retinacula" und Dennell (1933) als "coupling-hooks" bezeichnen und durch die jedes Pleopodenpaar miteinander verankert sein soll (siehe dazu auch Bousfield, 1973), die aber nach meinen Untersuchungen keinen effektiven Kopplungsmechanismus darstellen können wie etwa die Koppler der Thoracopoden bei den Copepoda (Manton, 1977; Kohlhage, 1983). Die Muskulatur der Pleopoden ist deutlich auf den Powerstroke ausgelegt; während für den Returnstroke nur jeweils ein Muskel am Protopoditen eines jeden Pleopoden angreift, wird der Powerstroke durch jeweils zwei Muskeln bewirkt (Vogel, 1986). 

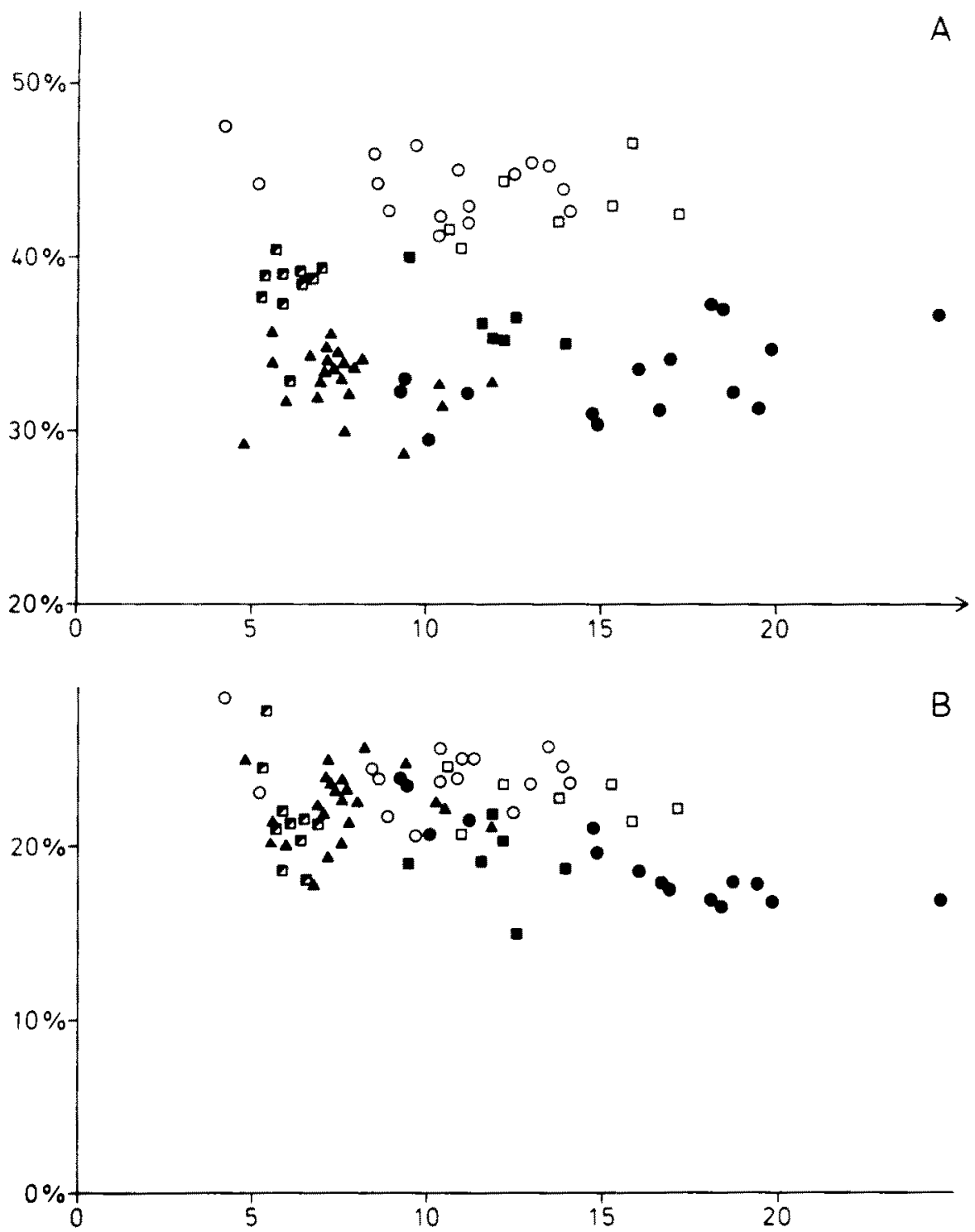

Abb. 3. Prozentuales Verhältnis von Abdomenlänge (A) bzw. Pleopodenlänge (B) zu Körperlänge (Ordinate) in Relation zur Körperlänge (Abszisse; in $\mathrm{mm}$ ). $\bullet$ Orchestia cavimana; $\backsim$ Talitrus saltator;

$₫$ Hyale nilssonii; $\square$ Gammarus locusta; $\bigcirc$ Gammarus pulex; $\bullet$ Corophium volutator

In der Relation Pleopodenlänge zu Gesamtkörperlänge unterscheiden sich die Arten nur wenig voneinander (Abb. 3 B). T. saltator und $O$. cavimana haben besonders mit zunehmender Körpergröße relativ etwas kürzere Pleopoden als die anderen untersuchten Arten (siehe auch Tab. 3); dieses zeigt sich besonders deutlich gegenüber den beiden Gammarus-Arten. Die Pleopoden wachsen bei allen untersuchten Arten annähernd isometrisch zur Körperlänge. 


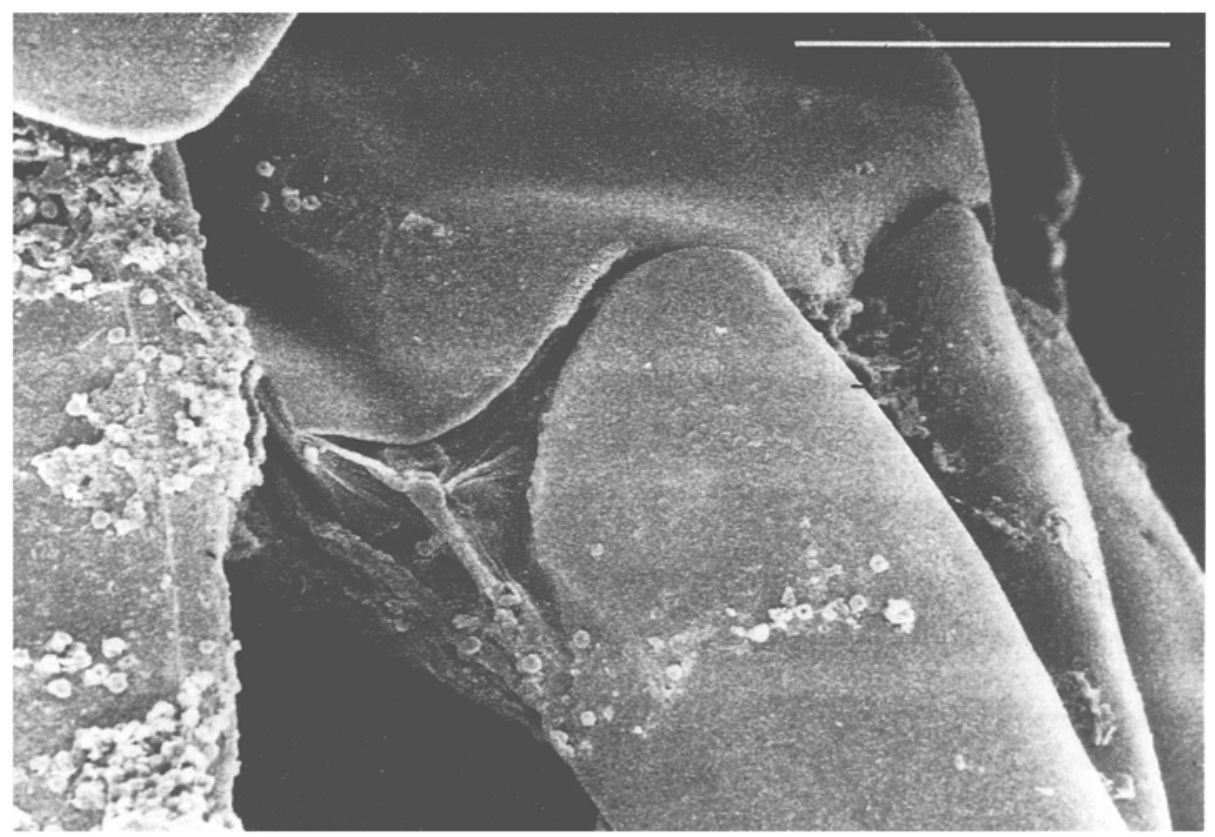

Abb. 4. Scharniergelenk zwischen Protopodit (oben) und Exo- und Endopodit (unten) eines Pleopoden von Hyale nilssonii. Maßstab 0,1 mm

Bei C. volutator sind die Protopoditen der Pleopoden plattenförmig verbreitert. Dadurch und durch die starke Befiederung der Exo- und Endopoditen, die auch bei den Talitridae und bei $G$. locusta recht stark ist - bei $G$. pulex weniger stark-, bieten die Pleopoden dem Wasser eine große Widerstandsfläche.

Die Gelenke zwischen Protopodit einerseits und Exo- bzw. Endopodit andererseits (Abb. 4) erlauben ein Abknicken der Äste caudad, aber nicht rostrad. Beim Powerstroke werden die Pleopoden passiv aufgrund des Strömungswiderstandes gestreckt, wobei die Widerstandsfläche weiter vergrößert wird. Die Kontraktion der entsprechenden Muskeln bewirkt eine zusätzliche Versteifung und ein Abspreizen der Pleopodenäste (Vogel, 1986). Beim Returnstroke knicken die Pleopodenäste dagegen - wiederum passiv - caudad ab und verkleinern so die Widerstandsfläche. Die beschriebenen Gelenke ermöglichen ferner eine mäßige Spreizung der Pleopoden.

Ahnlich sind die Borsten der befiederten Exo- und Endopoditen inseriert. Sie sitzen jeweils paarweise laterocaudal am proximalen Ende eines jeden Segmentes (Abb. 5). Beim Powerstroke werden sie passiv abgespreizt, wodurch die Widerstandsfläche der Pleopoden weiter vergrößert wird, beim Returnstroke der Pleopoden werden sie entsprechend V-förmig zusammengelegt, wie dieses von Kaestner (1967) und Bousfield (1973) beschrieben wird. So garantiert schon allein die mechanische Einlenkung der Pleopodenäste und der Borsten eine große Widerstandsfläche beim Powerstroke und eine wesentlich verkleinerte Widerstandsfläche beim Returnstroke der Pleopoden. 

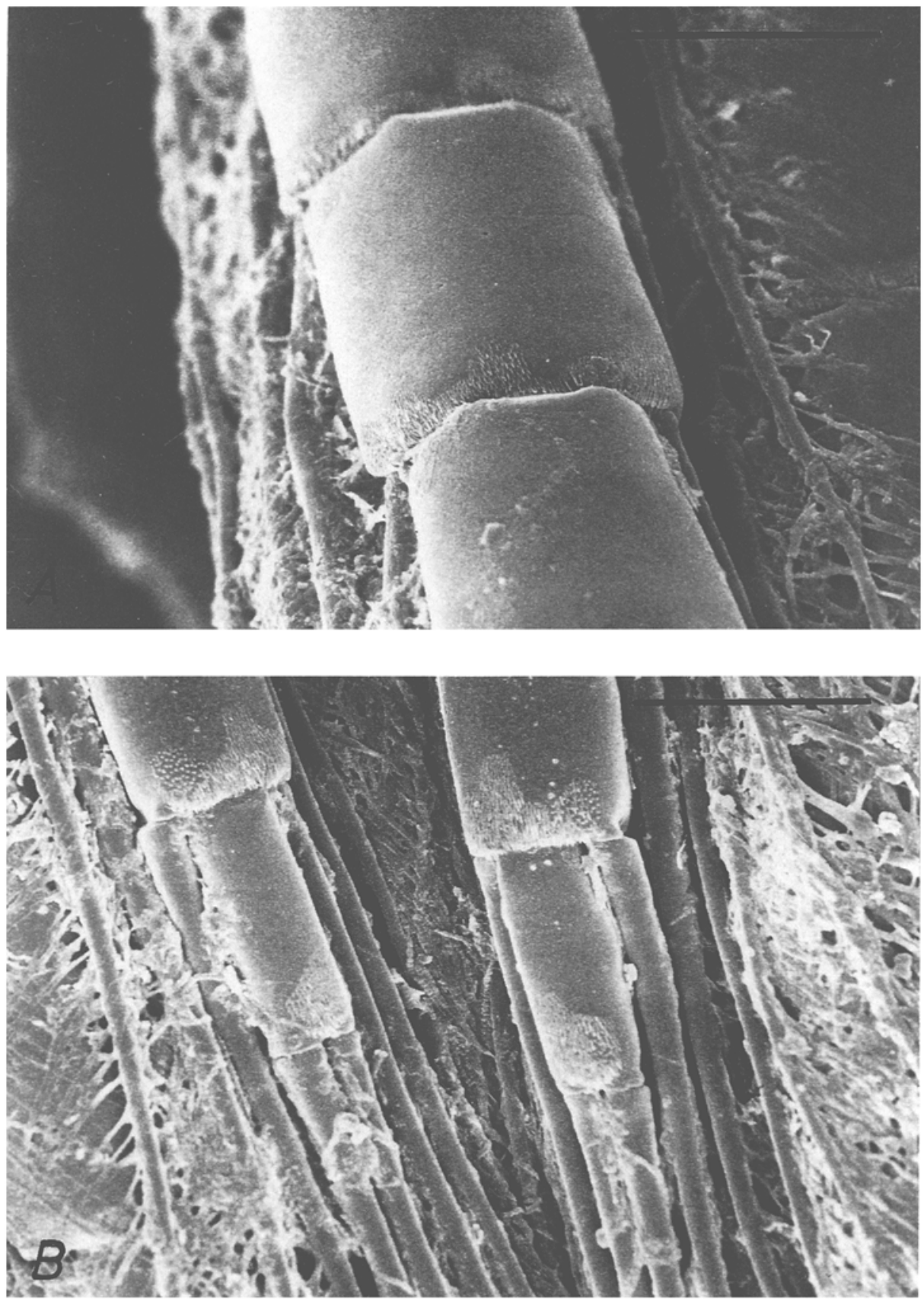

Abb. 5. Laterocaudale Insertion der Borsten an den einzelnen Segmenten der Exo- und Endopoditen eines Pleopodenpaares von Hyale nilssonii. A mittleres Segment eines Exopoditen; $B$ Endsegmente der Endopoditen. Maßstab $50 \mu \mathrm{m}$ 

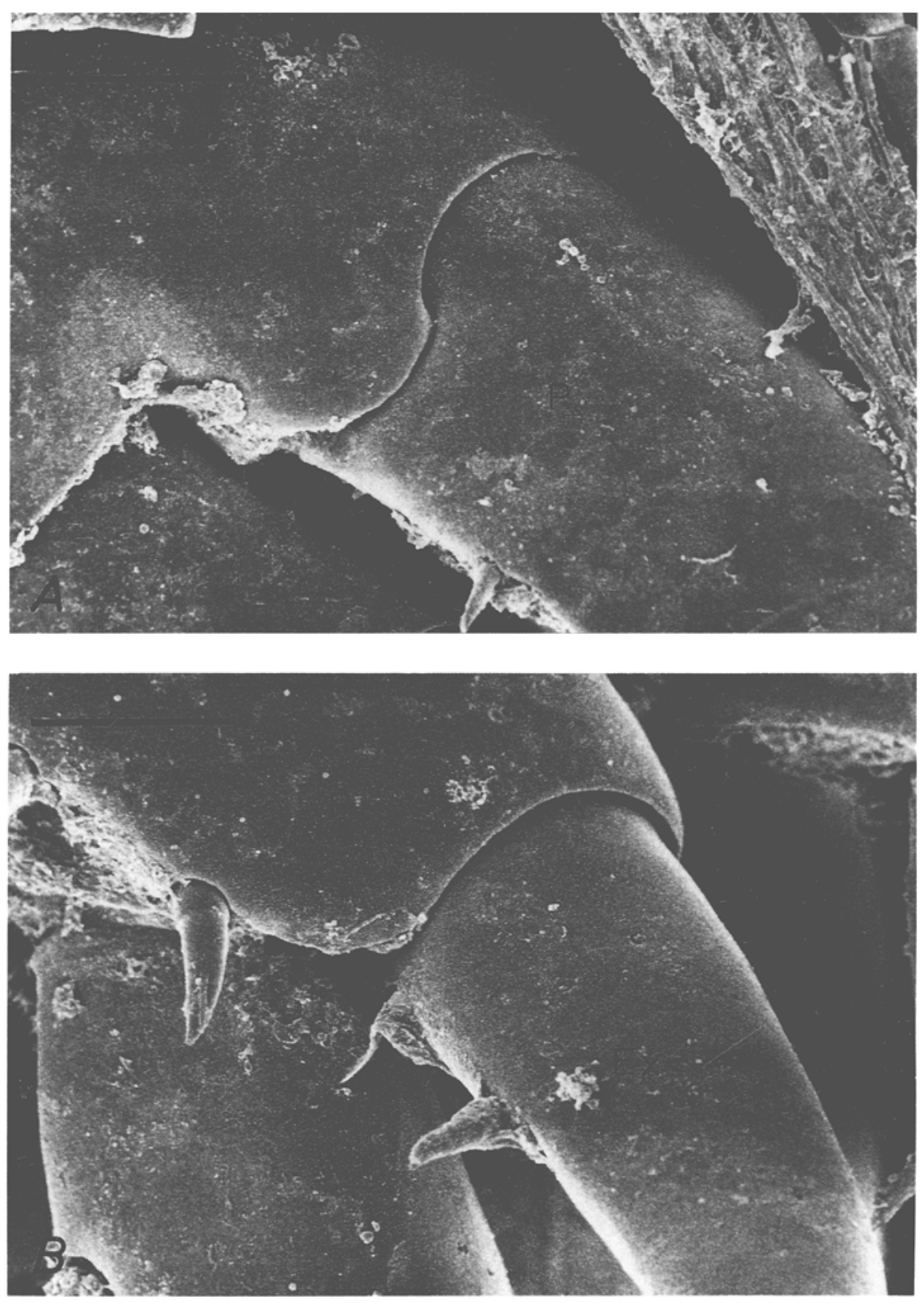

Abb. 6. A Gelenk zwischen erstem Urosomsegment und dem Protopoditen des rechten ersten Uropoden von Hyale nilssonii. B Gelenk zwischen Protopodit und Exopodit des rechten zweiten Uropoden von Hyale nilssonii. $\mathrm{p}=$ Protopodit; $\mathrm{Ex}=$ Exopodit. Maßstab 0,1 mm 


\section{Morphologie der Uropoden}

Die Uropoden sind ungeschützt an den hinteren Seitenecken der letzten drei Abdominalsegmente inseriert. Sie bestehen aus einem ungegliederten Protopoditen und zwei normalerweise eingliedrigen Ästen, dem Exo- und dem Endopoditen. Dieses Grundschema gilt für die ersten und zweiten Uropoden der untersuchten Arten fast ohne Einschränkung. Die dritten Uropoden sind dagegen unterschiedlich gestaltet. Nach Schellenberg (1942) unterliegen sie der Rückbildung.

Bei den beiden Gammarus-Arten sind die dritten Uropoden groß und zweiästig; der Exopodit ist außerdem zweigliedrig, allerdings ist das zweite Glied sehr klein. Rudimentär ist auch bei den Exo- und Endopoditen der ersten und zweiten Uropoden ein zweites Glied erhalten; dieses wurde von anderen Autoren bisher verneint $(z$. B. Schellenberg, 1942).

Die Talitridae zeigen eine generelle Längenreduzierung der dritten Uropoden; sie sind klein und einästig und tragen kräftige Terminaldornen. Dieses wurde von Bracht (1980b) als funktionelle Anpassung zum Anstemmen auf einer festen Unterlage interpretiert, was aber sicherlich keine Erklärung für die ebenfalls kleinen und einästigen dritten Uropoden von Corophium sein kann, die zudem noch verdickt sind.

Die Exo- und Endopoditen wie auch die Protopoditen der Uropoden sind so eingelenkt, daß sie - wie die Pleopodenäste - nur in einer Richtung abgeknickt werden können (Abb. 6), und zwar dorsad. Beim Abdomenschlag wird also mechanisch verhindert, daß die Uropoden ventrad umschlagen, wodurch die Effektivität des Abdomenschlages stark gemindert würde. Exo- und Endopodit sind - soweit beide vorhanden sind - nicht nebeneinander inseriert wie bei den Pleopoden, sondern schräg hintereinander, der Exopodit rostrad und etwas mehr distal. Neben dem dorsaden Abknicken ist eine Spreizung der Äste möglich, wie ich es auch schon für die Pleopodenäste beschrieben habe.

Die Muskulatur der Protopoditen zeigt keine auffallende Bevorzugung einer Bewegungsrichtung, Remotor und Promotor sind etwa gleich kräftig ausgebildet; weitere Muskeln dienen zur Abspreizung der Protopoditen, was für den Einsatz der Uropoden als Steuerungsorgan von Bedeutung ist (Vogel, 1986).

\section{Phänomenologie und Energetik des Schwimmens}

Zur Herausarbeitung der Unterschiede und Gemeinsamkeiten im Schwimmen der untersuchten Arten ist es neben der qualitativen Analyse erforderlich, die Schwimmbewegungen quantitativ zu erfassen und zu vergleichen, was dadurch erschwert wird, daß es sich beim Schwimmen nicht um einen dem Alles-oder-Nichts-Gesetz folgenden Vorgang handelt wie etwa beim Springen, sondern um eine in ihrem ganzen Verlauf sehr variable Bewegung (z. B. Schwimmrichtung, -geschwindigkeit, Einsatz des Abdomens). Abb. 7 zeigt einen Schwimmzyklus von $O$. cavimana als Sequenz von Filmbildern.

Den generellen Ablauf der Schwimmbewegung werde ich im folgenden stellvertretend für die Talitridae an $H$. nilssonii darlegen. Daran schließen sich interspezifischer und interfamiliärer Vergleich an. 

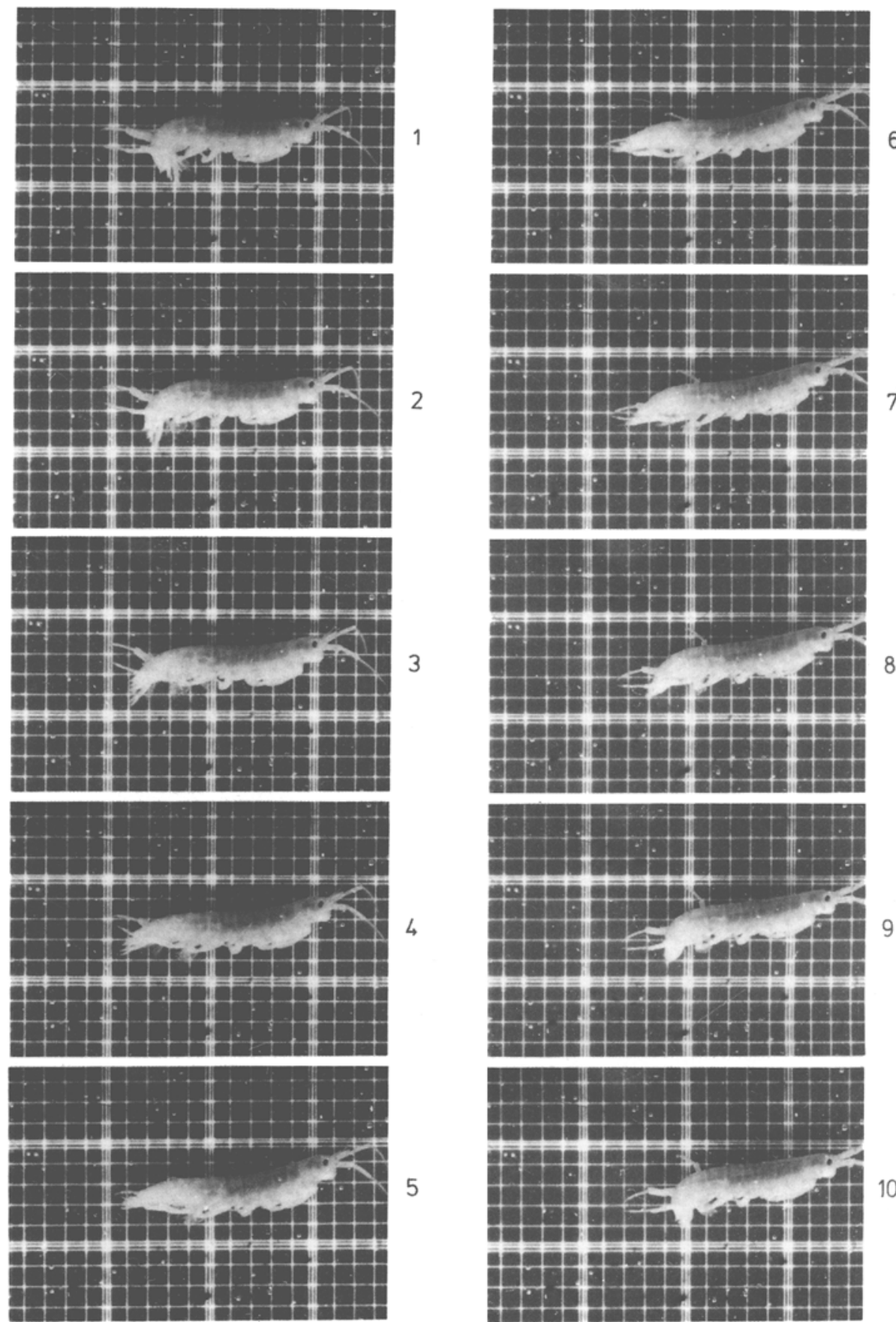

Abb. 7. Schwimmzyklus von Orchestia cavimana (Ausschnitt aus einer Hochfrequenzfilmaufnahme; zeitlicher Abstand zwischen den Einzelbildern: $6 \mathrm{~ms}$ ) 


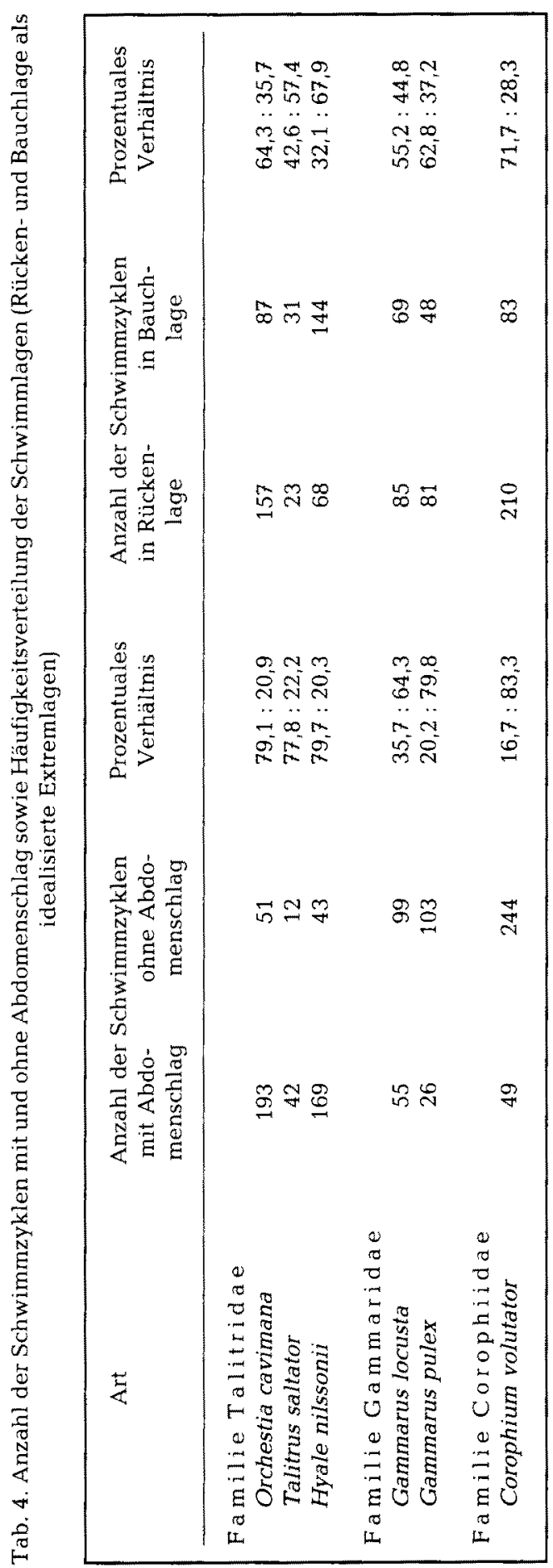




\section{Das Schwimmen von Hyale nilssonii}

Fast jeder Schwimmzyklus von $H$. nilssonii wird durch die Streckung des Abdomens eingeleitet (Tab. 4). Nur bei ca. $20 \%$ der filmisch aufgezeichneten Schwimmzyklen unterblieb der Abdomenschlag, der im Gegensatz zu O. cavimana und $T$. saltator bei $H$. nilssonii lediglich unterstützende Wirkung hat, denn Hauptantriebsorgan sind die Pleopoden. Noch während der Streckung des Hinterleibes (Abb. 8 und 9 ) beginnen die dritten Pleopoden und in Abständen von jeweils ca. 8 bis $10 \mathrm{~ms}$ die zweiten und danach die ersten Pleopoden mit caudad gerichtetem Schlag. Die zeitliche Aufeinanderfolge der drei Pleopodenpaare beim Powerstroke ist, verglichen mit $O$. cavimana und $T$. saltator (ca. $6 \mathrm{~ms}$ ), recht langsam. Dadurch bedingt werden häufig sogar schon die zweiten Pleopoden (und entsprechend auch die ersten) vom beginnenden Returnstroke der dritten Pleopoden und des Abdomens in ihrer Bewegung gestoppt. Der beim Powerstroke durch das Abdomen überstrichene Winkel (bis maximal ca. $50^{\circ}$ ) ist recht klein, und auch die erreichte Winkelgeschwindigkeit ist mit $2000 \%$ s oder $300 \mathrm{~min}^{-1}$ geringer als bei $O$. cavimana und $T$. saltator (vgl. Tab. 7). Die Pleopoden schlagen im Gegensatz zum Abdomen sehr kräftig und erreichen Winkelgeschwindigkeiten von bis zu $16500 \%$ oder $2750 \mathrm{~min}^{-1}$.

Da die Pleopoden bei Power- und Returnstroke in etwa gleich schnell bewegt werden, ist eine Verkleinerung der Widerstandsfläche durch Zusammenlegen der Pleopoden und Anlegen der Borsten beim Returnstroke, wie ich sie oben beschrieben habe, unbedingt notwendig, denn Power- und Returnstroke würden sich sonst in ihrer Wirkung aufheben. Beim Abdomen ist eine Verkleinerung der Widerstandsfläche nur in geringem Maße durch das Zusammenlegen und dorsade Abknicken der Uropoden möglich. Daher ist es angebracht, den Returnstroke des Abdomens um so langsamer durchzuführen, je größer der überstrichene Winkel beim Abdomenschlag ist, denn der Widerstand verhält sich proportional zum Quadrat der Geschwindigkeit; dieses zeigte sich besonders deutlich bei den großen $O$. cavimana.

H. nilssonii bevorzugt beim Schwimmen zu fast $70 \%$ die Bauchlage (Tab. 4). Beide Antennenpaare werden aufgrund des Strömungswiderstandes ventrocaudad abgeknickt. Die Pereiopoden 1 bis 4 werden nach vorn möglichst nahe an den Körper angelegt, die langen Pereiopoden 5 bis 7 nach hinten gestreckt bzw. durch den Strömungswiderstand in dieser Lage gehalten, was daraus ersichtlich ist, daß den Beschleunigungen und Abbremsungen des Körpers die Beine erst mit Verzögerung folgen. Die Uropoden (insbesondere die ersten) dienen neben der Steuerung offensichtlich auch der Lagestabilisierung, denn bei starken Abdomenschlägen werden sie weit gespreizt, während sie bei schwachen Abdomenschlägen zusammengehalten werden. Die Abdomenschläge folgen keineswegs dem Alles-oder-Nichts-Gesetz, sondern können in der Winkelgeschwindigkeit und im überstrichenen Winkel variieren.

\section{Interspezifischer und interfamiliärer Vergleich des Schwimmens}

Mittlere Körpergeschwindigkeit. Die mögliche Variationsbreite der mittleren Körpergeschwindigkeit kann als Maß für die Flexibilität beim Schwimmen angesehen werden. Die quantitative Auswertung der Filmaufnahmen ergab besonders für $G$. locusta, aber auch für $H$. nilssonii eine recht große Variationsbreite der Körper- 


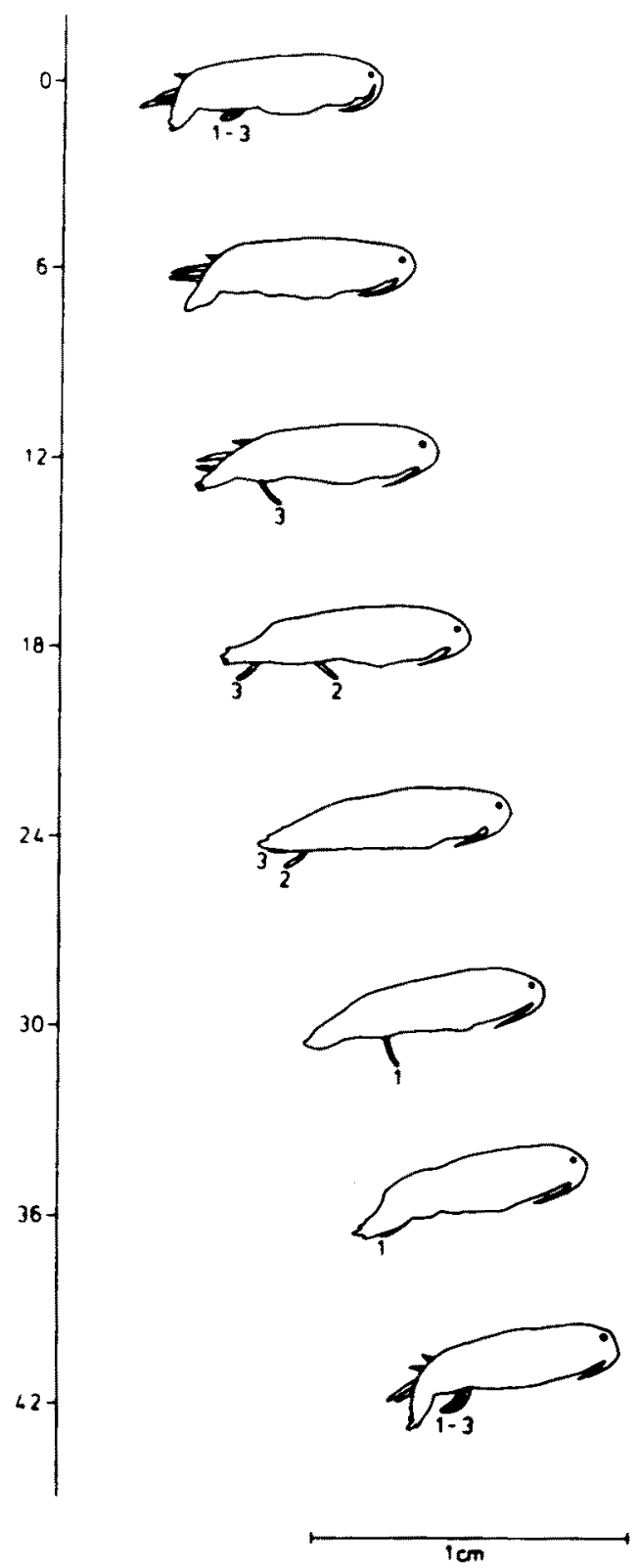

Abb. 8. Schwimmzyklus mit Abdomenschlag von Hyale nilssonii. Umzeichnung einer Hochfrequenzfilmaufnahme. 1-3 = Pleopoden; Ordinate: Zeit [ms]

geschwindigkeit (Tab.5). Die höchsten absoluten Körpergeschwindigkeiten werden ebenfalls von $G$. locusta und $H$. nilssonii erreicht. $O$. cavimana und $T$. saltator waren trotz ihrer Größe recht langsam, wobei die Angaben für $T$. saltator nicht repräsentativ sind, da nur drei quantitativ auswertbare Schwimmzyklen zur Verfügung standen. 

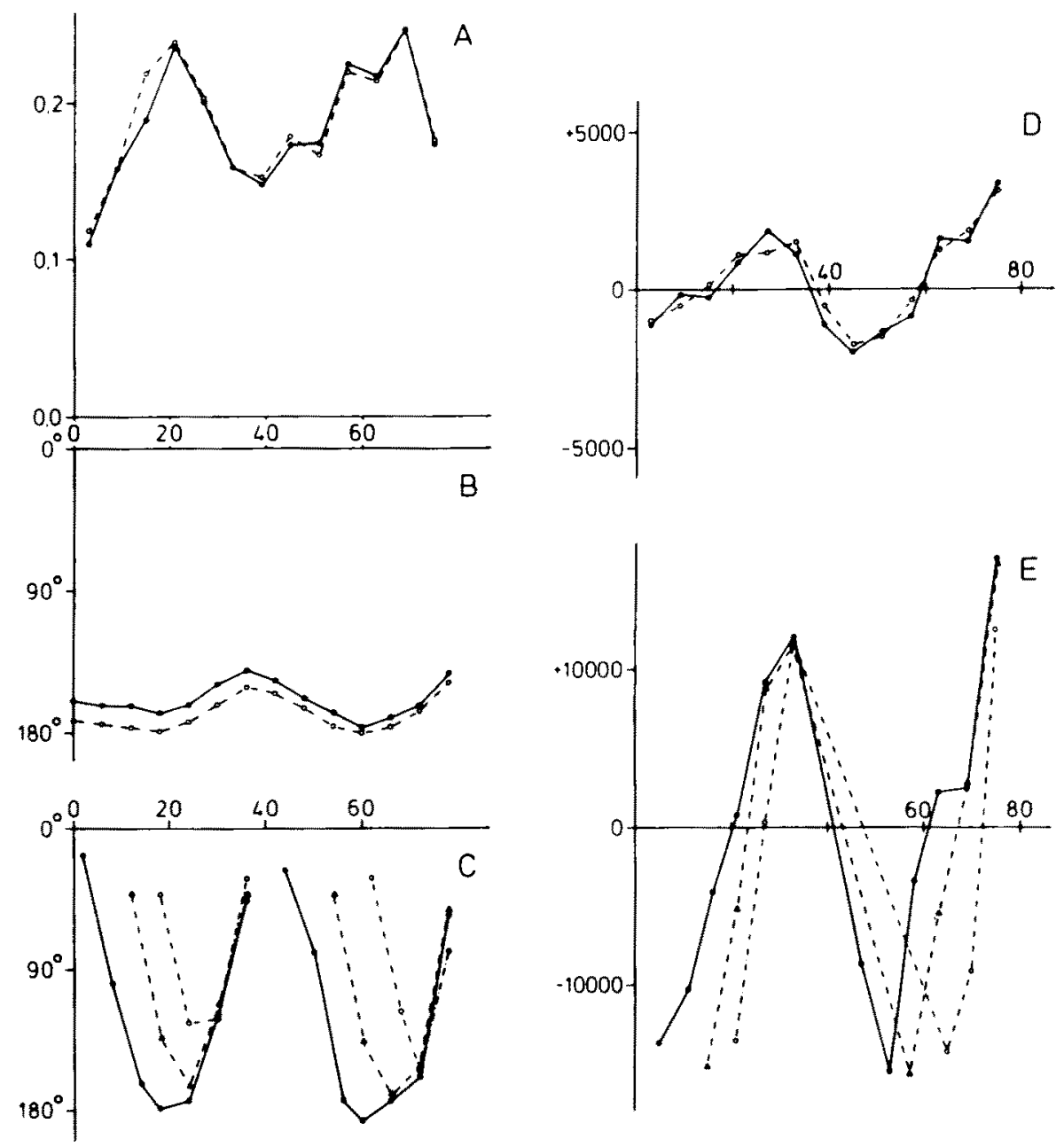

Abb. 9. Grafische Darstellung zweier Schwimmzyklen von Hyale nilssonii. Abszisse: Zeit [msec]. Ordinate: $A$ Körpergeschwindigkeit $\left[\mathrm{m} \cdot \mathrm{s}^{-1}\right]_{i} B$ Stellung der Abdomenachse zur Körperlängsachse $\left[^{\circ}\right]$; $C$ Stellung der Pleopodenachsen zur Körperlängsachse $\left[{ }^{\circ}\right]_{i} D$ Winkelgeschwindigkeit des Abdo-

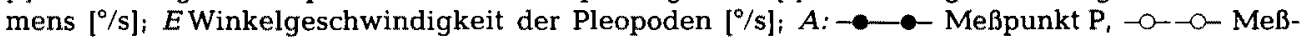
punkt $\mathrm{A}_{;} B$ und $D: \rightarrow-$ Meßachse $\overline{\mathrm{AU}}_{i} \multimap--0-$ Meßachse $\overline{\mathrm{AT}} ; C$ und $E:-\bullet-\bullet$ drittes Pleopodenpaar; - $\Delta-\cdot-$ - zweites Pleopodenpaar; $-O-\cdots \cdot-\infty$ erstes Pleopodenpaar

Um die Vergleichbarkeit trotz unterschiedlicher Körpergröße zu ermöglichen, relativierte ich die ermittelten Geschwindigkeitswerte zum einen bezüglich des Körpergewichtes, zum anderen bezüglich der Körper- bzw. Pleopodenlänge.

Die Variationsbreiten der Körpergeschwindigkeiten relativ zum Körpergewicht bieten ein etwas verschobenes Bild. $O$. cavimana und $T$. saltator zeigen bei dieser Darstellung wiederum recht niedrige Geschwindigkeiten, aber auch G. locusta erreicht nicht mehr so hohe Werte; er ist in seiner Leistungsfähigkeit mit $G$. pulex vergleichbar. Die größte Variationsbreite in dieser Gegenüberstellung zeigt $H$. nilssonii; diese Art ist 


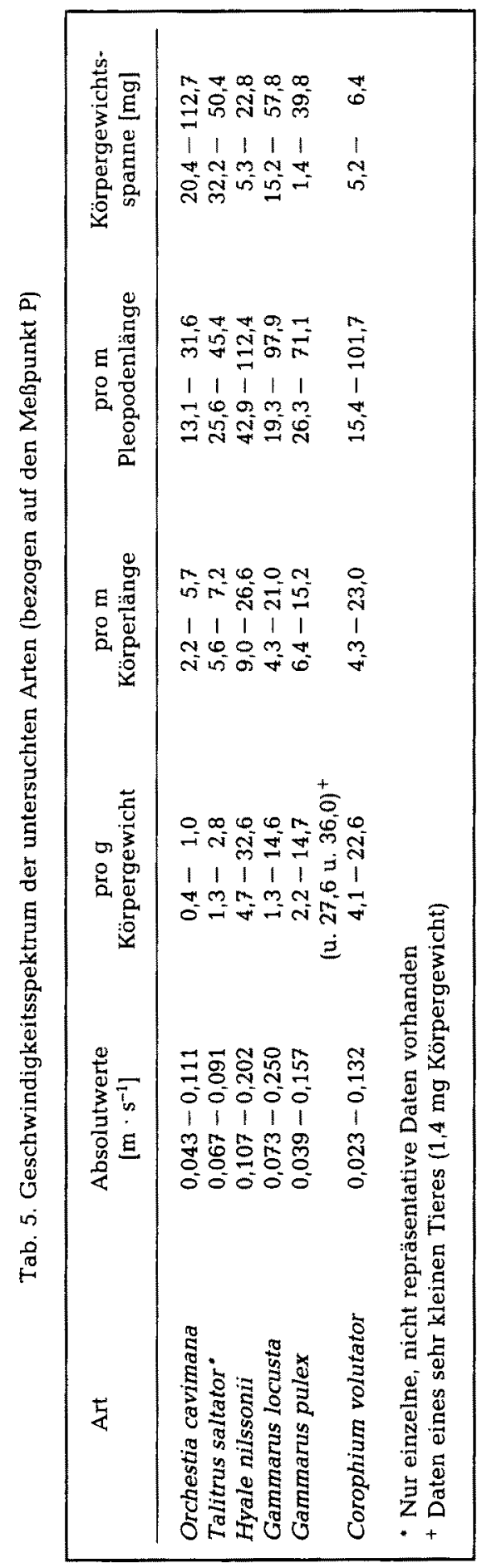


damit - relativ zum Körpergewicht - mit Abstand die schnellste Art, aber auch $C$. volutator erreicht noch recht hohe Werte.

In der Literatur zum Schwimmen verschiedener Tiere (siehe z. B. Kils, 1979) findet man häufig die sogenannte Körperlängengeschwindigkeit, also die Geschwindigkeit relativ zur Körperlänge, als Basisgröße für vergleichende Untersuchungen. Sie bietet eine bessere Vergleichsbasis als die Relativierung bezüglich des Körpergewichts. So wird bei dieser Darstellung deutlich, daß $C$. volutator relativ zu seiner Körperlänge (wobei die enorme Länge der zweiten Antennen nicht mit einbezogen ist) durchaus ein guter Schwimmer ist und in der Variationsbreite ein Bild ähnlich dem von G. locusta bietet. Die größte Variationsbreite und auch die insgesamt höchsten Werte zeigt wiederum $H$. nilssonii. G. pulex fällt in seinen Werten gegenüber G. locusta wieder etwas zurück und ist nun ungefähr gleichzusetzen mit $O$. cavimana. Eine ähnliche Klassifizierung der Arten erhält man bei der Betrachtung der Körpergeschwindigkeit relativ zur Pleopodenlänge und damit zur Länge der Hauptantriebsorgane.

Insgesamt erwies sich das Schwimmen von $H$. nilssonii, $G$. locusta und $C$. volutator als in der Geschwindigkeit flexibler gegenüber dem von $O$. cavimana, $T$. saltator und $G$. pulex, die bezüglich der untersuchten Körperparameter eine geringe Variationsbreite zeigten; auch in den absoluten Zahlenwerten liegen sie deutlich unter denen der erstgenannten Arten (mit Ausnahme von C. volutator).

S chwi m m y k lus länge. Die Schwimmzykluslänge ist sehr unterschiedlich; sie variiert zwischen $25 \mathrm{~ms}$ und $102 \mathrm{~ms}$. Allerdings lassen sich bestimmte Gesetzmäßigkeiten und auch artspezifische bzw. familienspezifische Unterschiede feststellen. So besteht generell die Tendenz zu zunehmender Schwimmzykluslänge bei zunehmendem Körpergewicht (Abb. $10 \mathrm{~A}$ ). Dabei zeigen die Talitridae kürzere Schwimmzykluslängen als die Gammaridae und als $C$. volutator. Bei $O$. cavimana und $T$. saltator (für letzteren ließ sich eine Regressionsgeradenberechnung nicht durchführen, da nur drei Werte zur Verfügung standen) stellte ich annähernd gleiche Schwimmzykluslängen fest, ebenso bei $G$. locusta, $G$. pulex und $C$, volutator. $H$. nilssonii nimmt bezüglich der Schwimmzykluslänge eine Mittelstellung zwischen diesen beiden Gruppen ein. Es liegt also gewissermaßen eine Trennung in Arten, die fast immer mit Abdomenschlag schwimmen (= Talitridae), und in Arten, die nur selten mit dem Abdomen schlagen (= Gammaridae und Corophiidae), vor, wobei $H$. nilssonii zu Recht die Mittelstellung einnimmt, da diese Art, wie ich schon oben feststellte, den Abdomenschlag nur unterstützend einsetzt.

Ein weiterer Parameter, der mit der Schwimmzykluslänge korreliert sein könnte, ist die Körpergeschwindigkeit. Die Vermutung, daß bei höherer Geschwindigkeit die Pleopoden in einem schnelleren Rhythmus geschlagen werden, die Schwimmzykluslänge also kürzer ist, wird durch die experimentellen Daten bestätigt. In Abb. $10 \mathrm{~B}$ wird deutlich, daß bei niedriger Körpergeschwindigkeit die Schwimmzykluslänge stark variieren kann, nicht jedoch bei hoher. Hohe Geschwindigkeiten erreichen nur $H$. nilssonii und $G$. locusta, wobei auch der letztere dazu den Abdomenschlag einsetzen muß. Ohne Abdomenschlag ist gerade bei G. locusta die Schwimmzykluslänge erheblich größer und die Geschwindigkeit geringer. Die Korrelation von Schwimmzykluslänge und Körpergeschwindigkeit wird durch die Regressionsgeraden deutlich (für $T$. saltator wurde keine Regressionsgeradenberechnung durchgeführt, es ist lediglich die Variationsbreite der Geschwindigkeiten angegeben). Insgesamt stellte ich bei den Gammarus-Arten, besonders bei $G$. locusta, eine größere Schwimmzykluslänge als bei 

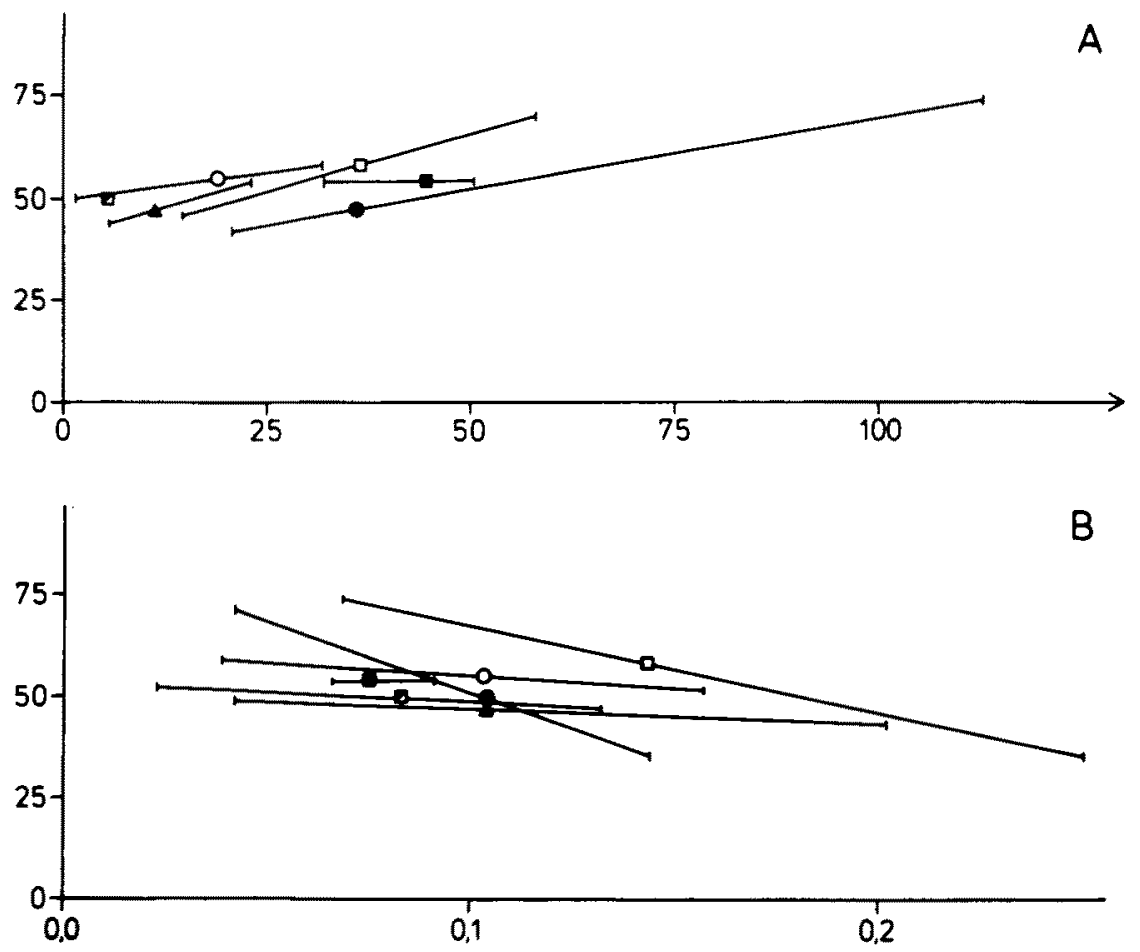

Abb. 10. Schwimmzykluslänge (Ordinate; in ms) in Relation zum Körpergewicht ( $A$; Abszisse; in $\mathrm{mg}$ ) und zur Körpergeschwindigkeit ( $B$; Abszisse; in $\mathrm{m} \cdot \mathrm{s}^{-1}$ ) unter Angabe der tatsächlichen Streuung (keine Regressionsgeradenberechnung für Talitrus saltator in $A$ und $B$ und für Corophium volutator in $A)$. Orchestia cavimana $\left(\mathrm{r}_{\mathrm{A}}=0,6548 ; \mathrm{r}_{\mathrm{B}}=-0,562\right)$; Talitrus saltator; $\Delta$ Hyale nilssonii $\left(\mathrm{r}_{\mathrm{A}}=0,4747 ; \mathrm{r}_{\mathrm{B}}=-0,250\right) ; \square$ Gammarus locusta $\left(\mathrm{r}_{\mathrm{A}}=0,4455 ; \mathrm{r}_{\mathrm{B}}=-0,631\right) ; 0$ Gammarus pulex $\left(r_{A}=0,3056 ; r_{B}=-0,177\right)$; Corophium volutator $\left(r_{B}=-0,536\right) ;\left(r_{A}\right.$ und $r_{B}=$ Korrelationsfaktoren)

den Talitridae (mit Ausnahme recht langsam schwimmender $O$. cavimana) fest. $C$. volutator unterscheidet sich in der Korrelation von Schwimmzykluslänge und Körpergeschwindigkeit dagegen kaum von den Talitridae, insbesondere $H$, nilssonii.

Abdomen - und Pleopodenschlag. Bei den Talitridae sind Abdomenund Pleopodenschlag weitestgehend miteinander gekoppelt. Die Gammaridae und auch die Corophiidae setzen dagegen den Abdomenschlag vor allem zum Abstoßen vom Untergrund und zum Wenden ein, wobei lediglich $G$. locusta in der Lage ist, den Abdomenschlag auch zum Schnellschwimmen zu nutzen. Nur bei dieser Art konnte ich eine deutliche Zunahme des beim Powerstroke durch das Abdomen überstrichenen Winkels mit zunehmender Körpergeschwindigkeit feststellen; der Abdomenschlag wird also in effektiver Weise zur Geschwindigkeitssteigerung eingesetzt. Bei $H$. nilssonii ist der überstrichene Winkel dagegen relativ konstant und damit unabhängig von der Geschwindigkeit. Einen ungezielten Einsatz des Abdomenschlages stellte ich bei $O$. cavimana und $T$. saltator, die beide, wie $H$. nilssonii, fast ausschließlich mit Abdomenschlägen schwimmen, fest.

Die Schwimmleistungen im Vergleich. Bei meinen Untersuchungen 
wurde deutlich, daß, je kleiner ein Tier ist, die Körperlängengeschwindigkeit um so größer ist, daß also die kleineren Tiere relativ zur Körperlänge schneller sind. Dieses gilt prinzipiell für alle Arten; allerdings erreicht $G$. locusta bei gleichen Körpergewichten größere Körperlängengeschwindigkeiten, jedoch nur unter Einsatz des Abdomenschlages. Ähnliches gilt für $H$. nilssonii und kleine $C$. volutator.

Um den - wenn auch kleinen - Unterschied zwischen den Familien, insbesondere zwischen den Gammaridae und den Talitridae, in der relativen Pleopodenlänge mit in die vergleichende Betrachtung einzubeziehen, wählte ich eine Bezugsgröße, die mit der technischen Größe PS/kg oder $\mathrm{kW} / \mathrm{kg}$ vergleichbar ist. Als Leistungseinheit dient unter starker Vereinfachung - die Pleopodenlänge. Ich betrachte also das Produkt aus Körpergeschwindigkeit und $\frac{\text { Pleopodenlänge }}{\text { Körpergewicht }}$ in Relation zur Körperlänge.

Da die Pleopoden fast isometrisch zur Körperlänge und damit negativ allometrisch zum Körpergewicht wachsen, wird das Verhältnis von Pleopodenlänge zu Körperge-

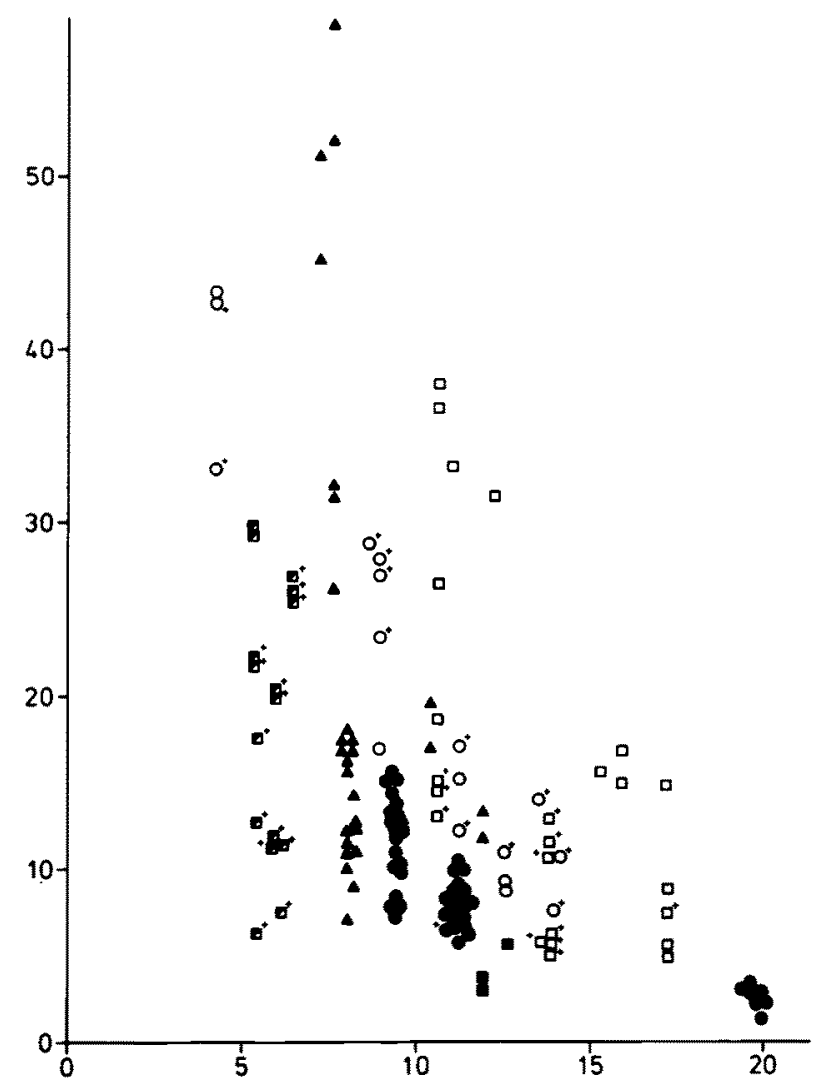

Abb. 11. [Körpergeschwindigkeit $\left.\frac{\text { Pleopodenlänge }}{\text { Körpergewicht }}\right]\left(\right.$ Ordinate; in $\left.\mathrm{m}^{2} \cdot \mathrm{s}^{-1} \cdot \mathrm{kg}^{-1}\right)$ in Relation zur Körperlänge (Abszisse; in $\mathrm{mm}$ ). Erläuterungen siehe Text. + Schwirnmzyklus ohne Abdomenschlag; • Orchestia cavimana; $\bullet$ Talitrus saltator; $\triangle$ Hyale nilssonii; $\square$ Gammarus locusta; O Gammarus pulex; 1 Corophium volutator 
wicht mit zunehmender Größe ungünstiger. Ein gutes Verhältnis von Pleopodenlänge zu Körpergewicht ist also ebenso wie eine höhere Körpergeschwindigkeit positiv zu werten. Das Produkt aus Körpergeschwindigkeit und $\frac{\text { Pleopodenlänge }}{\text { Körpergewicht }}$ bietet dadurch eine Vergleichsbasis für die Schwimmgüte, die in der Relation zur Körperlänge betrachtet werden muß, da größere Tiere schon aufgrund der oben beschriebenen morphologischen Gegebenheiten benachteiligt sind.

Abb, 11 verdeutlicht, daß $G$. locusta fast durchweg höhere Werte erzielt als etwa gleich große Vertreter der anderen Arten. Lediglich kleine $H$. nilssonii erreichen ähnlich gute Werte, große dagegen sind mit $G$. pulex und kleinen $O$. cavimana vergleichbar. $T$. saltator und große $O$. cavimana lassen nur ein mäßiges Schwimmvermögen erkennen. Für die Talitridae ergibt sich also eine deutlichere Abnahme in der Schwimmgüte mit zunehmender Körpergröße als für die Gammaridae. Die Daten der Corophiidae sind nur eingeschränkt vergleichbar, da sich der Bau der Pleopoden dieser Amphipoda doch sehr von dem der Gammaridae und Talitridae unterscheidet.

Abschließend betrachte ich die erzielten kinetischen Energien, also die Energiebeträge, die für die Translationsbewegung benötigt werden (bezogen auf $1 \mathrm{~kg}$ Körpergewicht; Abb. 12). Während die meisten Versuchstiere nur kinetische Energien von maximal ca. $10^{-2} \mathrm{~J} / \mathrm{kg}$ erreichen, erzielt $G$. locusta bis zu dreifach höhere Werte, und auch kleine $H$. nilssonii liegen mit bis zu doppelt so hohen Werten deutlich über dem

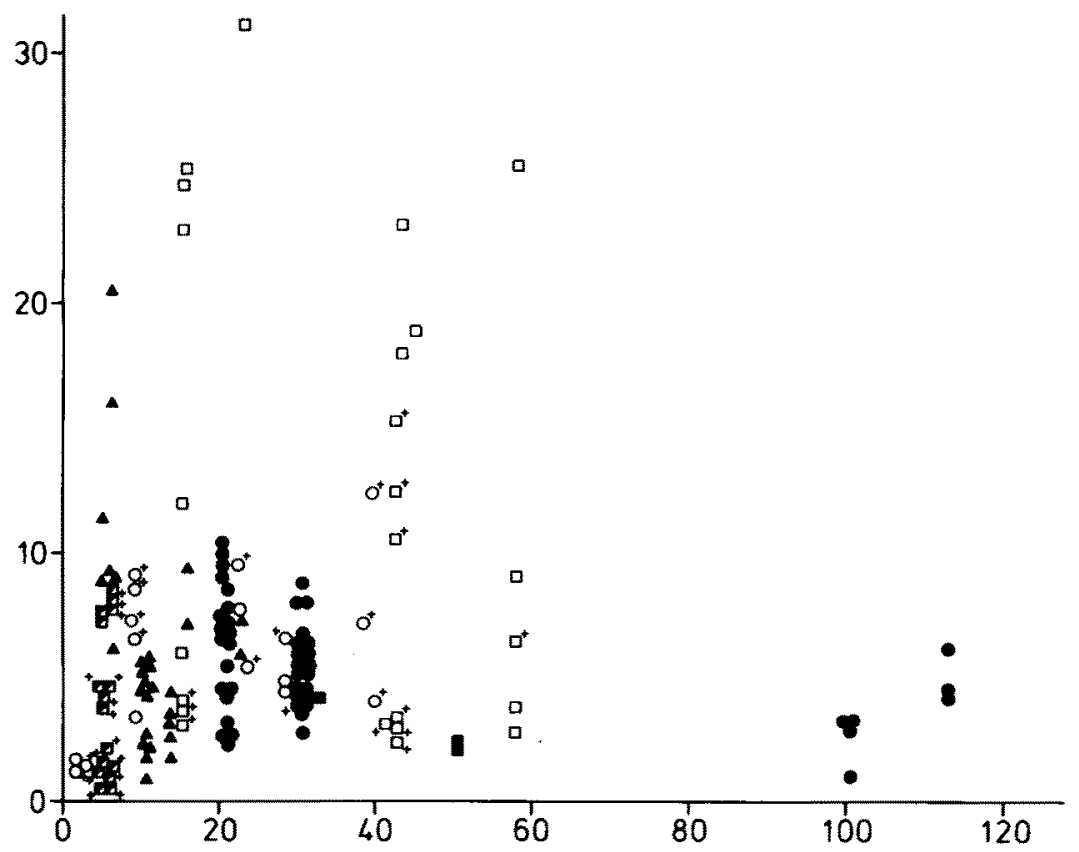

Abb. 12. Kinetische Energie pro $\mathrm{kg}$ Körpergewicht (Ordinate; in $10^{-3} \mathrm{~J} / \mathrm{kg}$ ) in Relation zum Körpergewicht (Abszisse; in mg). + Schwimmzyklus ohne Abdomenschlag; - Orchestia cavimana; - Talitrus saltator; 4 Hyale nilssonii; $\square$ Gammarus locusta; O Gammarus pulex; Corophium volutator 
Durchschnitt. Hier erweisen sich also ebenfalls $G$. locusta und kleine $H$. nilssonii als die besten Schwimmer.

\section{DISKUSSION}

\section{Das Schwimmen als Anpassung an den Lebensraum}

Wie die obigen Ausführungen immer wieder verdeutlichen, sind Gammarus locusta und Hyale nilssonii die besten Schwimmer unter den untersuchten Arten. Berücksichtigt man den Nachteil, den Corophium volutator aufgrund seines Habitus hat, so zeigt auch diese Art durchaus gutes Schwimmvermögen. Ein weniger guter Schwimmer ist Gammarus pulex, der aber im Schnitt doch noch etwas leistungsfähiger ist als Orchestia cavimana und Talitrus saltator.

Die untersuchten Arten lassen sich in drei ökologische Gruppen einteilen:

- wattbewohnende Arten der Gezeitenzone,

- Süßwasser-Arten in fließenden Gewässern und

- (semi-)terrestrische Arten.

$G$. locusta und $H$. nilssonii kommen vergesellschaftet in den Algenbeständen der Gezeitenzone des Wattenmeeres vor, z. B. auf Fucus vesiculosus. Sie sind dort stark wechselnden Umweltbedingungen ausgesetzt, die eine flexible Anpassung erfordern. Dieses spiegelt sich in der Variabilität des Schwimmens wider, wie ich sie oben dargelegt habe, aber auch in den hohen maximal erreichten Werten (Körpergeschwindigkeit, Körperlängengeschwindigkeit, kinetische Energie pro kg Körpergewicht). In diesem Lebensraum sind die Tiere häufig erheblichen Wasserkräften ausgesetzt; die Krebse müssen auch bei stärkeren Strömungen in der Lage sein, sich fortzubewegen.

Dieses gilt in ähnlicher Weise auch für $C$. volutator, wobei dieser Krebs aber den Vorteil hat, daß er zu einer halb-sedentären Lebensweise übergegangen ist. C. volutator baut Höhlen und ernährt sich im Umkreis seiner Wohnstätte. Allerdings konnte ich auch große Mengen von $C$, volutator beobachten, wie sie bei einsetzender Ebbe in den Prielen schwammen, wo durchaus stärkere Strömungen auftreten können. C. volutator muß also - zumindest kurzfristig - in der Lage sein, einer Verdriftung entgegenwirken zu können, wozu eine gewisse Anpassungsfähigkeit, wie sie die Variationsbreite der Körpergeschwindigkeit andeutet, vonnöten ist. Die geringe Ausdauer, die diese Krebse zeigen, ist mit der halb-sedentären Lebensweise erklärbar, die nicht erfordert, daß $C$. volutator in der Lage ist, größere Entfernungen schwimmend zu überbrücken.

G. pulex lebt als Süßwasser-Art im Pflanzenbewuchs klarer fließender Gewässer. Er ist also ständig einer Strömung ausgesetzt, die aber in aller Regel schwächer ist als Strömungen in der Gezeitenzone des Wattenmeeres. Die Gefahr der Verdriftung ist für einen Amphipoden in diesem Lebensraum also nicht so groß wie im Wattenmeer. Bei schwächeren Strömungen ist G. pulex durchaus in der Lage, eine mögliche Verdriftung durch Schwimmen zu kompensieren, wie auch Hughes (1970) feststellte. G. pulex ist also an diesen speziellen Lebensraum in bezug auf das Schwimmvermögen in genügender Weise angepaßt.

$O$. cavimana und $T$. saltator als (semi-)terrestrische Vertreter der Gammaridea sind in ihrem normalen Lebensraum nicht darauf angewiesen, gut schwimmen zu können, da sie nur in Ausnahmefällen ins Wasser gelangen. Für diese Krebse ist eher ein gutes Sprungvermögen von Vorteil, das aber andere morphologische Anpassungen erfordert 
als das Schwimmen. So war aufgrund der Lebensweise zu erwarten, daß diese beiden Arten schlechteres Schwimmvermögen zeigen würden als etwa $H$. nilssonii und $G$. locusta, was durch die experimentellen Daten bestätigt werden konnte. Jedoch zeigte es sich, daß kleine $O$. cavimana (von $T$. saltator liegen keine entsprechenden Daten vor) noch durchaus in ihrer Leistungsfähigkeit mit $G$. pulex vergleichbar sind und daß diese erst mit zunehmender Körpergröße relativ schlechter wird, daß also insbesondere die großen Individuen, die morphologisch in bezug auf die Anpassung an die (semi-) terrestrische Lebensweise voll ausgebildet sind, zum Schwimmen nicht mehr so gut geeignet sind. Diese Schlußfolgerung wird auch durch den ineffektiven Einsatz des Abdomenschlages bestätigt, der energetisch wesentlich aufwendiger ist als eine Sequenz von Pleopodenschlägen, da eine wesentlich größere Masse bewegt werden muß.

\section{Der Abdomenschlag beim Schwimmen als homologe Bewegung zur Extension des Abdomens beim Sprung der Talitridae}

Bevor ich anhand der von Remane $(1955,1961)$ aufgestellten Homologiekriterien auf die Homologisierbarkeit des Schwimmens mit Abdomenschlag mit dem Springen der Talitridae eingehe, diskutiere ich zunächst die Untersuchungen von Bracht (1978, unveröffentlicht; $1980 \mathrm{a}, 1980 \mathrm{~b}$ ) zum Springen der Talitridae im Vergleich mit meinen Befunden zum Schwimmen.

Bracht untersuchte das Springen der Talitridae mit Hilfe der Hochfrequenzkinematographie $(1000$ bis $2500 \mathrm{~B} / \mathrm{s})$ und der Stroboskopmethode. Er unterscheidet drei Sprungphasen, die Sprungvorbereitung, den Absprung und die Phase nach dem Absprung. Zur Vorbereitung eines Sprunges wird der Thorax stark gebeugt (Buckelbildung), und die Uropoden werden bei eingeschlagenem Abdomen weit gespreizt auf den Untergrund aufgesetzt. Während des Absprunges treten zwei Beschleunigungsschübe auf. Der erste (im Mittel ca. $60 \%$ der Gesamtbeschleunigung) kommt durch eine plötzliche Streckung des Thorax in Richtung der Körperlängsachse zustande, der zweite (ca. $40 \%$ ) durch "blitzschnelle" Extension des Abdomens. Die Abdomenstreckung reicht also zum Springen allein ebensowenig aus wie der Abdomenschlag zum Schwimmen.

Außer für die auch von mir untersuchten Talitridae gibt Bracht (1980b) Daten zum Sprung von $O$. gammarella und $O$. platensis, die beide an Kiesstränden der Nordsee im Bereich der Hochwasserlinie vorkommen. Die wichtigsten Sprungdaten der fünf Arten sind in Tab. 6 zusammengefaßt. Aus den Daten geht klar hervor, daß T. saltator das beste Sprungvermögen aufweist. Nicht wesentlich schlechter sind $O$. gammarella und $O$. platensis. Dagegen erreichen $O$. cavimana und $H$. nilssonii nur 55 bis $70 \%$ (je nachdem, welche Daten man zugrunde legt) der Leistung von $T$. saltator. Etwas höhere Werte für O. cavimana gibt Bracht in seinen früheren Arbeiten (1978, unveröffentlicht; 1980a) an; danach würde diese Art eine Mittelstellung zwischen den beiden anderen OrchestiaArten und $H$. nilssonii einnehmen. Allerdings beruhen diese Daten jeweils auf nur wenigen Sprüngen.

Bracht versuchte, die unterschiedlichen Sprungleistungen unter morphologischen Gesichtspunkten zu erklären (wobei ich den Begriff "Leistung" hier und im folgenden nicht im physikalischen Sinne gebrauche, sondern darunter die "Leistungsfähigkeit", also das Sprung- bzw. Schwimmvermögen, verstehe), und kam zu dem Ergebnis, daß 
F. Vogel

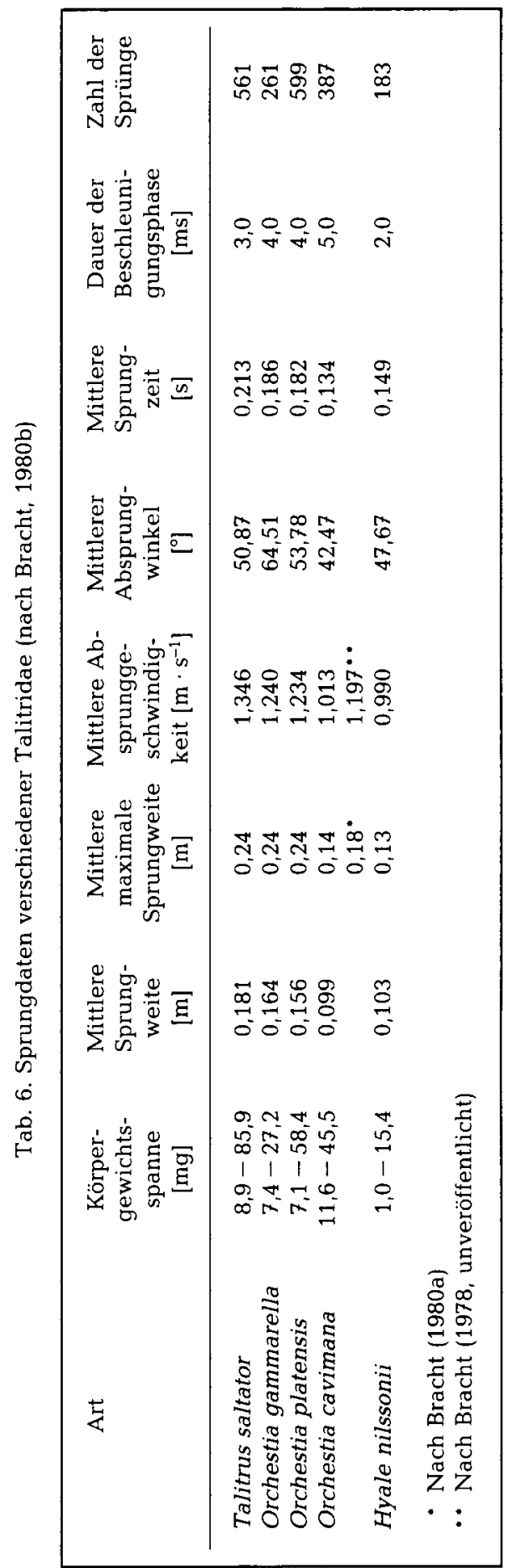


innerhalb einer Art die schwereren Individuen zwar weniger weit springen - sowohl relativ zur Körperlänge als auch, wenn auch weniger deutlich, absolut -, aber daß durchschnittlich schwerere Arten weiter springen als durchschnittlich leichtere. Seine Einteilung in leichte bis schwere Arten ist jedoch fraglich. Es ist sicherlich richtig, daß H. nilssonii eine durchschnittlich kleinere Art ist, jedoch lassen sich die anderen vier Arten nicht eindeutig als schwerer oder leichter klassifizieren.

Das Ergebnis, daß kleine Individuen relativ zur Körpergröße weiter springen als größere, daß sie also das bessere Sprungvermögen zeigen, stimmt mit meinen Befunden bezüglich des Schwimmvermögens überein; selbst absolut ist die Sprunghöhe kleinerer Tiere noch etwas größer als die größerer Tiere (siehe Bracht, 1980b). Dieses ist auch verständlich, denn das Körpergewicht wirkt sich besonders in Luft (beim Springen) nachteilig aus. Im Wasser (beim Schwimmen) spielen neben dem Gewicht die Gesamtwiderstandsfläche des Körpers und damit der Reibungswiderstand eine Rolle.

Die Betrachtung der von Bracht $(1980 \mathrm{~b})$ beschriebenen Sprungleistungen der Talitridae unter ökologischen Gesichtspunkten führt zu dem Schluß, daß das Sprungvermögen in Relation zum Grad der Adaptation an die terrestrische Lebensweise zu sehen ist. T. saltator ist zweifelsfrei die am besten an die terrestrische Lebensweise adaptierte Art der untersuchten Talitridae. Dementsprechend zeigt er auch das größte Sprungvermögen. O. gammarella und $O$. platensis, die ähnliche Biotope bevorzugen, unterscheiden sich kaum in ihrer Sprungfähigkeit. Zu erwarten wäre, daß $O$. cavimana, die ebenfalls auf steinigen Substraten oberhalb des normalen Wasserpegels lebt, nicht sehr viel geringere Leistungen zeige als die beiden anderen Orchestia-Arten. Dieses ist jedoch nicht der Fall: Das deutlich schlechtere Sprungvermögen von $O$. cavimana läßt sich vielleicht mit der gegenüber den Biotopen von $O$. gammarella und $O$. platensis weniger exponierten Lage ihres Biotops erklären. Dadurch könnte das Fluchtvermögen gemindert sein. Ähnlich geringe Sprungleistungen zeigt $H$. nilssonii; relativ zum Körpergewicht ist sein Sprungvermögen sogar als etwas schlechter zu bewerten, da die Versuchstiere dieser Art durchschnittlich wesentlich kleiner waren als $O$. cavimana und kleinere Tiere im Schnitt höhere Leistungen (Sprungweite, Absprunggeschwindigkeit; absolut und relativ zur Körpergröße) zeigen als größere, wie oben erläutert.

Vergleicht man nun die Sprungdaten mit dem Ergebnis der Untersuchungen zum Schwimmen der Talitridae, so fällt sofort auf, daß die Art mit dem geringsten Sprungvermögen, $H$. nilssonii, das beste Schwimmvermögen zeigt und dieses bei nur unterstützendem Einsatz des Abdomenschlages, der aber doch recht effektiv ist. Umgekehrt ist $T$. saltator als ausgezeichneter Springer nur ein sehr mäßiger Schwimmer. O. cavimana ist weder ein guter Springer noch ein guter Schwimmer, nimmt also eine Mittelstellung ein; allerdings zeigen kleine $O$. cavimana relativ gute Schwimmleistungen. Auch Dahl (1946) sieht in Orchestia, speziell O. platensis, einen besseren Schwimmer als in Talitrus. Schon aus diesem groben Leistungsvergleich läßt sich eine deutliche Abhängigkeit zwischen Schwimm- und Sprungvermögen ablesen. Diese beiden Leistungskriterien stehen in einem reziproken Verhältnis zueinander. Es bestehen also zwei gegenläufige, aber gegenseitig bedingte Entwicklungsreihen, die gemeinsam in Korrelation mit der Adaptation an die terrestrische Lebensweise zu sehen sind. Indirekt erfüllt dieses die Forderung des dritten Hauptkriteriums der Homologie, des Kriteriums der Stetigkeit bzw. Kontinuität.

Wie Vogel (1986) zeigen konnte, besteht die dorsale Muskulatur, die für die 
Extension des Abdomens zuständig ist, fast ausschließlich aus dem paarigen dorsalen Längsmuskelstrang, der, obwohl er sich aus fünf Muskeln zusammensetzt, als eine Einheit zu betrachten ist. Es ist also nur ein Muskelsystem vorhanden, das eine effektvolle Extension des Abdomens sowohl beim Schwimmen als auch beim Springen ermöglicht, was der Forderung des ersten Hauptkriteriums der Homologie, des Kriteriums der Lage, entspricht. Daß die Muskeln auch beim Springen direkt wirken, daß also kein Energiespeichermechanismus wie etwa bei den Schnellkäfern, den Elateridae (Kaschek, 1984), oder den Springschwänzen, den Collembola (Christian, 1979), vorhanden ist, beweist die Tatsache, daß bei allen untersuchten Talitridae Ermüdungserscheinungen bei aufeinanderfolgenden Sprüngen festgestellt werden konnten (Bracht, $1980 b)$. Diese zeigen sich in einer Abnahme der Sprungweite sowie der Sprungfrequenz bis zur Thanatose. Wäre ein Energiespeichermechanismus vorhanden, der dem Allesoder-Nichts-Gesetz folgte, so dürfte wohl die Sprungfrequenz mit zunehmender Sprungzahl abnehmen, aber nicht die Sprungweite.

Ein prinzipieller Unterschied zwischen dem Springen und dem Schwimmen zeigt sich in der Geschwindigkeit der Abdomenextension. Dieser Unterschied ist nicht auf die unterschiedlichen Medien (Luft, Wasser) zurückzuführen. Ich konnte nämlich sowohl von $O$. cavimana als auch von $H$. nilssonii Abdomenschläge im Wasser filmisch festhalten, die in ihrem Verlauf der Abdomenextension beim Springen ähnlich sind - allerdings mit dem Unterschied, daß die Tiere sich nicht von einer Unterlage abstießen, sondern frei im Wasser schwammen. Sie erreichten Winkelgeschwindigkeiten von 5- bis 10fach höheren Werten als beim "normalen" Abdomenschlag während des Schwimmens. Der Returnstroke des Abdomens erfolgt wieder mit "normaler" Geschwindigkeit. Bemerkenswert dabei ist, daß sich das Tier aufgrund seiner Trägheit während des Abdomenschlages fast nicht von der Stelle bewegt; dieser "blitzschnelle" Abdomenschlag ist also für das Schwimmen völlig ungeeignet.

Wie kommt dieser Unterschied im Geschwindigkeitsverlauf zustande? Vermutlich handelt es sich um ein etwas unterschiedliches Zusammenspiel der Flexor- und Extensormuskeln, wie schon Bracht (1980b) vermutete. Während beim Schwimmen mit Abdomenschlag die Flexoren und Extensoren sich abwechselnd kontrahieren und somit eine harmonische Abdomenbewegung bewirken, dürften bei der "blitzschnellen" Extension des Abdomens zunächst sowohl Flexoren als auch Extensoren kontrahiert sein, die Streckmuskeln allerdings nur mäßig, weshalb das Abdomen trotz ihrer Anspannung in dieser Sprungvorbereitungsphase in der eingeklappten Stellung verbleibt. Bei plötzlicher Erschlaffung der Flexoren und gleichzeitiger, weiterer Kontraktion der Extensoren wird das Abdomen - wie zu beobachten ist - innerhalb weniger Millisekunden aus der flektierten in die extendierte Stellung gebracht. Aus der Vorspannung der Extensoren gegen die Kontraktion der Flexoren resultiert demnach die Wirkungsverstärkung, die für die "blitzschnelle" Extension beim Sprung notwendig ist. Das Kriterium der speziellen Qualität (der Abdomenextension) - zweites Hauptkriterium der Homologie - ist also nicht hundertprozentig erfültt. Jedoch reicht der beschriebene Unterschied im Zusammenspiel der Extensor- und Flexormuskeln auch nicht aus, um die beiden Bewegungsweisen als qualitativ eindeutig unterschiedlich ansehen zu können.

Insgesamt können also der Abdomenschlag beim Schwimmen und die plötzliche Extension des Abdomens beim Springen durchaus als homologe Bewegungen betrach- 
tet werden, zumal diese Schlußfolgerung auch durch die Anwendung der drei Hilfskriterien (Remane, 1961) gestützt wird; denn die Ubberlegungen sind sicherlich nicht nur auf die Familie der Talitridae zu beschränken, sondern können durchaus auf andere nahe verwandte Amphipoda wie z. B. die Gammaridae ausgedehnt werden (erstes Hilfskriterium). So findet sich das Merkmal des Schwimmens mit Abdomenschlag auch innerhalb dieser Familie, und auch das Springen wurde für eine Gammarus-Art, $G$. duebeni, beschrieben (Segerstråle, 1946); quantitative Aussagen liegen jedoch leider nicht vor, so daß hier vergleichende Betrachtungen nicht möglich sind.

Die Gammaridae und Talitridae bilden ferner eine morphologisch-anatomisch recht einheitliche Gruppe, was den Anforderungen des zweiten Hilfskriteriums entspricht. Umgekehrt bestehen große morphologische und funktionelle Unterschiede (drittes Hilfskriterium) zu anderen Krebsen wie etwa den Decapoda, insbesondere den Langschwanzkrebsen, die beim Schwimmen den Abdomenschlag als Fluchtmechanismus einsetzen - allerdings mit dem Unterschied, daß der Powerstroke nicht die Abdomenextension, sondern die Abdomenflexion ist, das Tier also dabei rückwärts schwimmt.

\section{SCHLUSSBEMERKUNGEN}

Innerhalb der Familie der Talitridae ist jede Übergangsform von litoraler bis terrestrischer Lebensweise verwirklicht. Bezüglich der Morphologie der Pleopoden schreibt Dahl (1977), daß selbst die supralitoralen Formen der Gattungen Talitrus, Orchestia und Talorchestia normal gebaute Pleopoden haben. Jedoch läßt sich das so verallgemeinernd nicht sagen. Barnard (1969) sieht gerade den Verlust von Pleopoden als Kennzeichen zum einen der sedentär lebenden Caprellidea, zum anderen aber auch der terrestrischen Arten der Talitridae an, und Gerstaecker \& Ortmann (1901) leiten aus der Verkleinerung der Pleopodenpaare bei den "Orchestiidae" das ". . Unvermögen, sich schwimmend fortzubewegen . . ", ab. Bousfield (1973) betrachtet umgekehrt kräftig gebaute Pleopoden als Merkmal röhrenbewohnender, grabender Arten oder auch pelagischer Arten wie z. B. Phronimopsis tumida (Familie Hyperiidae), die als Tiefseeart große Vertikalwanderungen durchführt und dementsprechend starke Pleopoden mit kräftiger Muskulatur besitzt (Vester, 1900).

Die terrestrischen Arten der Talitridae, insbesondere die in Lauberde tropischer und subtropischer Wälder lebenden, zeigen alle Stadien der Pleopodenreduktion (Hurley, 1968): So besitzen Orchestia lesliensis und Talitrus sylvaticus noch zwei Paare voll entwickelter Pleopoden, bei $O$. rubroannulata ist nur das erste Pleopodenpaar normal entwickelt, und $O$. patersoni weist lediglich noch Reduktionsknospen auf. Nach Stephensen (1938) sollen die Pleopoden bei $O$, patersoni sogar vollständig reduziert sein.

Aber nicht nur äußerlich erkennbare Reduktionen der Pleopoden lassen die Adaptation an die terrestrische Lebensweise erkennen, sondern auch die Muskulatur noch vorhandener Pleopoden oder Pleopodenglieder ist betroffen. So stellte Schellenberg (1938) bei Arten der Gattung Orchestoidea fest, daß die Pleonsegmente ". . . muskelfreie Pleopodenstiele, an denen nur eine kleine Knospe als Überbleibsel eines Pleopodenastes entspringt . . .", tragen; die Stiele der ersten Pleopoden wiesen noch Andeutungen eines Muskels auf. Und auch Bowers (1964) beobachtete bei $O$. californiana und $O$. corniculata, daß die Pleopoden für das Schwimmen relativ unbrauchbar seien und daß die Tiere daher verstärkt den Abdomenschlag einsetzten. 
Es zeigt sich also, daß die Pleopodenreduktion nicht ausschließlich als phylogenetische Entwicklungsreihe zu betrachten ist, sondern insbesondere als Adaptation an den jeweiligen Biotop, an die spezielle Lebensweise. So findet man sie genauso gut bei den Caprellidea (Barnard, 1969; siehe oben) wie auch z. B. bei dem Einsiedlerkrebs Pagurus pollicarus, der adult nur noch auf der linken Abdomenseite Pleopoden besitzt, die den Atemwasserstrom im Schneckengehäuse in Bewegung halten (Bent \& Chapple, 1977); die Larvalformen dieses Krebses haben dagegen normal bilateralsymmetrisch angelegte Pleopoden.

Die von mir untersuchten Talitridae wiesen im Bau der Pleopoden kaum Unterschiede zu den Gammaridae auf, und auch Hessler (1982) sieht die Pleopoden von Talitrus saltator und Gammarus pulex als gleich gebaut an. Daraus läßt sich schließen, daß T. saltator, obwohl diese Art von den von mir untersuchten die am besten an die terrestrische Lebensweise angepaßte ist, noch nicht so weit vom Wasser als Lebensraum gelöst ist wie andere Talitridae, z. B. der als Gewächshaus-Flohkrebs bekannte Talitroides alluaudi. Die morphologische Adaptation der Pleopoden kann aber nicht nur als Anpassung an die Lebensweise betrachtet werden, sondern muß auch in den Gesamthabitus eingeordnet werden, wobei Gesamthabitus und Lebensweise allerdings eng korreliert sind (vgl. Corophium volutator: plattenförmige Verbreiterung der Protopoditen der Pleopoden in Korrelation mit dem dorso-ventral abgeflachten Körperbau).

Bei den Amphipoda betrachtet Barnes (1968) allgemein die Pleopoden als Hauptantriebsorgan beim Schwimmen und sieht im Abdomenschlag lediglich einen Mechanismus, sich vom Substrat abzustoßen und so eine relativ hohe Anfangsgeschwindigkeit zu erreichen. Dennell (1933) geht sogar so weit, die Möglichkeit, einen Abdomenschlag in effektiver Weise ausführen zu können, eher als negatives Kriterium bezüglich des Schwimmvermögens einer Art zu werten. "This flexion probably prevents an amphipod such as Gammarus locusta from swimming in a regular manner, as a curved body travelling through a dense medium such as water will tend to follow a curved path." Die von ihm untersuchte Art Haustorius arenarius (Familie Haustoriidae) sieht er als “. . . beautifully constructed for swimming and burrowing . .." an, da sie weitestgehend die Möglichkeit verloren hat, den Körper zu beugen und zu strecken, also einen Abdomenschlag auszuführen, und bezeichnet das Schwimmen von $H$. arenarius, obschon diese Art langsamer sein soll als G. locusta (genaue Zahlenangaben gibt er nicht), als "graceful".

Hier muß man sich die Frage stellen, nach welchen Kriterien das Schwimmvermögen eines Tieres zu beurteilen ist. Ein sicherlich vorrangiger Bewertungsmaßstab ist die erreichbare Geschwindigkeit (absolut und relativ zur Körperlänge), die beim Schwimmen mit Abdomenschlag durchaus höher sein kann als beim reinen "Pleopodenschwim. men" (Tab. 7), wie Kils (1979) bei Euphausia superba (Euphausiacea - Leuchtgamelen) feststellte; er bezeichnet das Schwimmen mit Abdomenschlag als "Schwanzschwim. men" im Gegensatz zum "Pleopodenschwimmen", wobei als weiterer Unterschied die Umkehrung der Schwimmrichtung hinzukommt. Der diskontinuierliche Antrieb durch Abdomenschläge kann also durchaus Vorteile gegenüber dem kontinuierlicheren Antrieb durch die Pleopoden haben, zumal wenn er als Fluchtmechanismus dient. Energetisch dürfte er aufwendiger sein. Aber auch das muß nicht immer für diskontinuierliche Antriebe gelten, wie Weihs (1977) mit Hilfe eines analytischen Modells nachwies. Webb (1979) ermittelte für das Schwanzschwimmen von Orconectes virilis einen 
Wirkungsgrad von immerhin $58 \%$, für das Pleopodenschwimmen von $68 \%$, also Werte, die nach seinen Angaben weit über denen von Fischen liegen (10 bis $20 \%$ Wirkungsgrad). Auch beim Vergleich der Körpergeschwindigkeiten schneiden Fische nicht so gut $a b$, wie man vielleicht erwarten würde. Nach Nursall (1962) erreichen sie zwar absolut gesehen wesentlich höhere Geschwindigkeiten als beispielsweise die Amphipoda und auch als andere Crustacea (vgl. Hargreaves, 1981, und Tab. 7); bezieht man die Geschwindigkeit jedoch auf die Körperlänge (Körperlängengeschwindigkeit), so zeigen die untersuchten Gammaridea fast duchweg höhere Werte und damit größere Leistungsfähigkeit, auch wenn die Fische hydrodynamisch günstiger gebaut zu sein scheinen.

Schon Hertel (1967) stellte jedoch fest, daß es ". . . keine allgemeingültige Bestform des Rotationskörpers und keine 'Laminarform' schlechthin, sondern immer nur eine für einen engen Reynolds-Bereich günstige Form ..." gibt (die Reynolds-Zahl gibt das Verhältnis der Trägheitskräfte zu den Zähigkeitskräften an), und auch Nachtigall (1977) schrieb, daß neben der Körperform auch die absoluten Dimensionen des Körpers eine entscheidende Rolle spielen. Diese werden um so bedeutungsvoller, je kleiner ein Körper ist oder - anders ausgedrückt - je kleiner die Reynolds-Zahl ist. Für Amphipoda liegt die Reynolds-Zahl im Bereich von 1 bis 100, also in einem Bereich, in dem Trägheitskräfte und Zähigkeitskräfte sich in etwa die Waage halten. Für Euphausia superba ermittelte Kils (1979) einen Reynolds-Bereich von 50 bis 850 und - interessanterweise - fast gleiche Widerstandsbeiwerte ( $c_{w}$-Werte) als Angabe des Strömungswiderstandes für das Pleopodenschwimmen $\left(c_{w}=0,31\right)$ und das Schwanzschwimmen $\left(c_{w}=0,33\right)$, was auf ähnliche Wirkungsgrade hindeutet. Dieses ist also ein weiterer Hinweis dafür, daß es energetisch nicht ungünstiger sein muß, mit Hilfe eines diskontinuierlichen Antriebs zu schwimmen.

Arthropoden zeigen allgemein einen evolutiv alten Typ der Bein-Koordination, die kreuzweise Verschaltung (von Holst, 1935). Der Phasenunterschied zwischen zwei kontralateralen Beinen muß bei den Crustacea zwar nicht genau $1 / 2$ betragen, sondern kann auch auf $1 / 3$ verringert sein - dieses hängt von der Gesamtzahl der beteiligten Beine $a b$; das synchrone Bewegen kontralateraler Beine ist jedoch als Spezialisierung auf eine bestimmte Fortbewegungsweise zu sehen.

So schlagen die Portunidae, die Schwimmkrabben, ihr fünftes Beinpaar beim Schwimmen synchron (von Holst, 1935); dieses Beinpaar ist auch morphologisch gut an die Schwimmbewegung angepaßt. Spirito (1972) beobachtete bei Callinectes sapidus (Portunidae) ebenfalls eine Gleichschaltung des fünften Beinpaares beim Schwimmen, während aber gleichzeitig die zweiten bis vierten Beine gemäß der normalen, kreuzweisen Verschaltung bewegt wurden. Kühl (1933) beobachtete, daß die Schwimmbeine bei den Portunidae beim langsamen Rückwärtsschwimmen auch alternierend geschlagen werden können. Eine ähnliche Spezialisierung nur eines Beinpaares zeigen die Winkerkrabben (Ocypodidae). Bei primitiven Arten ist das Winken der großen Schere (und phasenverschoben auch der kleinen Schere) mit in das Verschaltungsschema der Schreitbeine einbezogen (Altevogt, 1972), d. h., beim Winken muß das Tier die entsprechenden Schreitbeine mit bewegen, muß also zwangsläufig laufen; bei höher entwickelten Arten zeigt sich die zunehmende Loslösung des Winkens vom Laufen, was als phylogenetisches Merkmal betrachtet werden kann.

Eine kontralaterale Synchronisierung der Schwimmbeine, der Pleopoden, ist bei den Amphipoda gegeben. Obwohl sich dieses Verschaltungsschema schon bei primiti- 


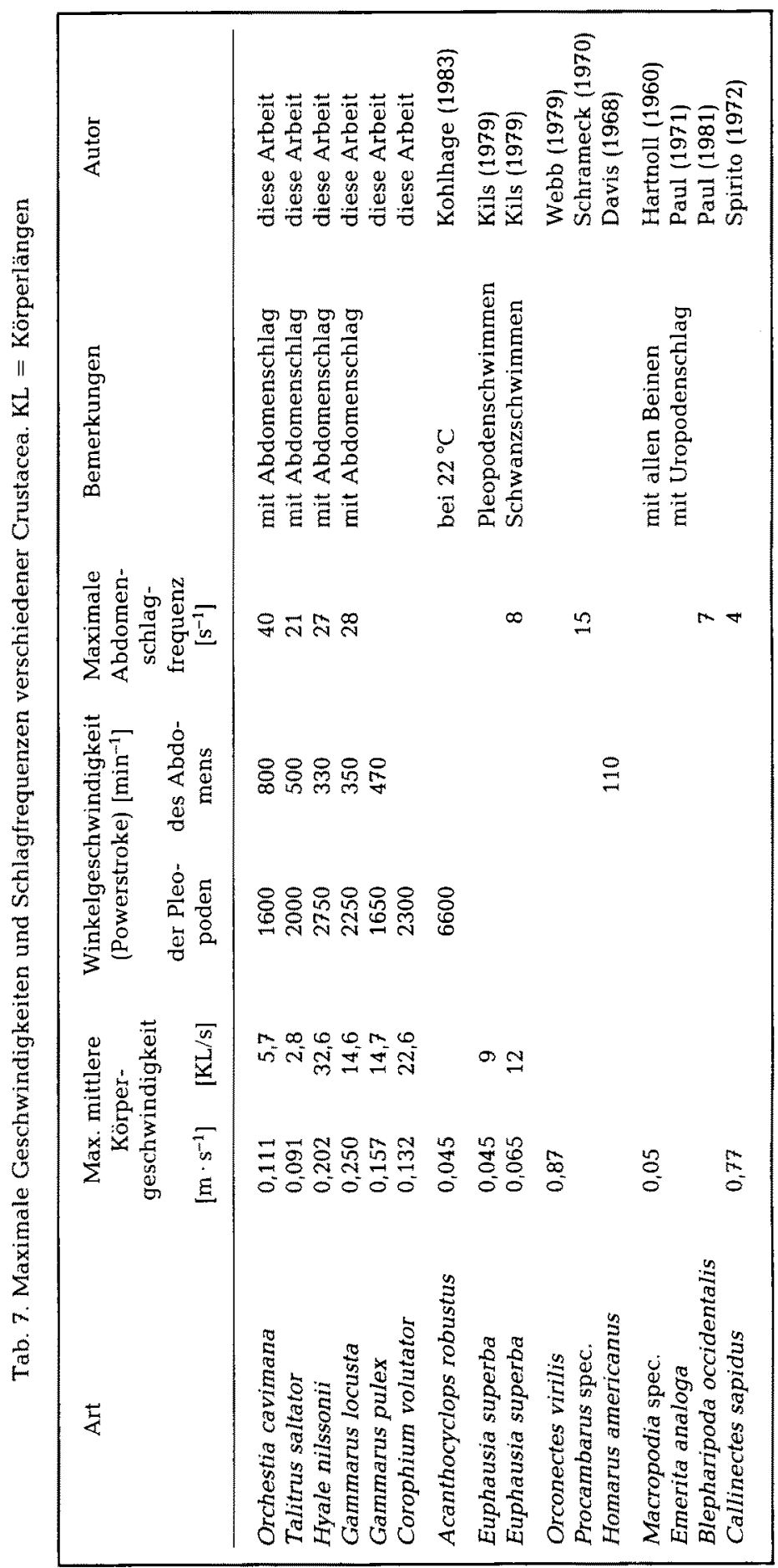


ven Crustacea wie Limulus oder Artemia findet, muß es nach von Holst (1935) als abgeleitet betrachtet werden. Auch die primitive Art Anaspides tasmaniae (Syncarida) zeigt beim Schwimmen schon einen metachronalen Schlagrhythmus der Pleopoden (MacMillan et al., 1981), jedoch besteht in erster Linie eine ipsilaterale Koordination der Beine, d.h., die Beine jeweils einer Seite bilden eine Einheit, während die beiden Seiten nur lose aneinander gekoppelt sind. Ähnliches stellte Hartnoll (1970) bei der nur schlecht an das Schwimmen angepaßten Art Homola barbata (Familie Dromiidae) fest.

Barr \& Smith (1980) kamen durch ihre Untersuchungen zum Schwimmen der Wassermilbe Limnocharus americana zu dem Schluß, daß “... diagonal phase synchrony represents an ancestral coordination pattern which is less efficient than the more derived method of coordination by transverse phase synchrony". Die kontralaterale Synchronisierung findet man also bei spezialisierten, gut an ihre Lebensweise angepaßten Formen wie z. B. den Amphipoda oder auch den Copepoda (Kohlhage, 1983), die zudem noch eine mechanische Kopplung der kontralateralen Beine entwickelt haben.

Danksagung. Herrn Professor Dr. R. Altevogt danke ich für die großzügige Unterstützung meiner Arbeit und die stete Hilfs- und Gesprächsbereitschaft sowie für die Bereitstellung von Kameraausrüstung und Filmmaterial, Mein Dank gilt auch Herrn Dr. N. Kaschek und Herrn Dr. J. Sitterle für technische Hilfe bei meinen zum Teil recht aufwendigen Aufnahmeverfahren.

\section{LITERATUR}

Abele, L. G., 1982. Biogeography. In: The biology of Crustacea. Ed. by D. E. Bliss. Acad. Press, New York, 1, 242-304.

Altevogt, R., 1971. Unterklasse Höhere Krebse. In: Grzimeks Tierleben. Hrsg. von B. Grzimek. Kindler, Zürich, 1, 468-506.

Altevogt, R., 1972. Physiological inter-relationships of display and locomotion in fiddler crabs: an evolutionary aspect. - J. mar. biol. Ass. India 14, 456-467.

Arendse, M. C., 1980. Non-visual orientation in the sandhopper Talitrus saltator (Mont.). - Neth. J. Zool. 30, 535-554.

Barnard, J. L., 1969. The families and genera of marine gammaridean Amphipoda. - Bull. U.S, natn. Mus. 271, 1-535.

Barnes, R. D., 1968. Invertebrate zoology. Saunders, Philadelphia, 743 pp.

Barr, D. \& Smith, B. P., 1980. Stable swimming by diagonal phase synchrony in arthropods. - Can. J. Zool. 58, 782-795.

Bent, S. A. \& Chapple, W. D., 1977. Simplification of swimmeret musculature and innervation in the hermit crab, Pagurus pollicarus, in comparison to macrurans. - J. comp. Physiol. 118, 61-73.

Bousfield, E. L., 1973. Shallow-water Gammaridean Amphipoda of New England. Comstock, Ithaca, $312 \mathrm{pp}$.

Bousfield, E. L. \& Howarth, F. G., 1976. The cavernicolous fauna of Hawaiian lava tubes. 8. Terrestrial Amphipoda (Talitridae), including a new genus and species with notes on its biology. - Pacif. Insects 17, 144-154.

Bowers, D. E., 1964. Natural history of two beach hoppers of the genus Orchestoidea (Crustacea: Amphipoda) with reference to their complemental distribution. - Ecology 45, 677-696.

Bracht, G., 1980a. The jump of Orchestia cavimana Heller, 1865 (Crustacea, Amphipoda, Talitridae). - Experientia 36, 56-57.

Bracht, G., $1980 \mathrm{~b}$. Vergleichende phänomenologische und energetische Studien zum Sprung der Talitridae (Crustacea, Amphipoda). Diss,, Münster, 68 pp.

Christian, E., 1979. Der Sprung der Collembolen. - Zool. Jb. (Allg. Zool. Physiol. Tiere) 83, 457-490.

Dahl, E., 1946. Undersökningar över Oresund. XXIX. The Amphipoda of the sound. - Acta. Univ. Lund. (Av. 2) 57 (6), 1-51. 
Dahl, E., 1977. The amphipod functional model and its bearing upon systematics and phylogeny.Zool. Scr. 6, 221-228.

Davis, W. J., 1968. Quantitative analysis of swimmeret beating in the lobster. - J. exp. Biol. 48, $643-662$.

Dennell, R., 1933. The habits and feeding mechanism of the amphipod Haustorius arenarius Slabber. - J. Linn. Soc. (Zool.) 38, 363-388.

Dudich, E., 1927. Neue Krebstiere in der Fauna Ungarns. - Archvm balaton., Bpest 1, 343-387.

Gerstaecker, A. \& Ortmann, A. E., 1901. Die Klassen und Ordnungen der Arthropoden: Crustacea: Malacostraca: Amphipoda: Flohkrebse. - Bronn's Kl. Ordn. Tierreichs 5 (Abt. 2, Hälfte 2), $279-543$.

Hargreaves, B. R., 1981. Energetics of crustacean swimming. In: Locomotion and energetics in Arthropoda. Ed. by C. F. Herreid \& C. R. Fourtner. Plenum Press, New York, 453-490.

Hartnoll, R. G., 1960. Swimming in spider crabs of the genus Macropodia. - Nature, Lond. 185, 181.

Hartnoll, R. G., 1970. Swimming in the dromiid crab (Homola barbata) - Anim. Behav. 18, 588-591.

Heinze, K., 1932. Fortpflanzung und Brutpflege bei Gammarus pulex L. und Carinogammarus roeselii Gerv. - Zool. Jb. (Allg. Zool. Physiol. Tiere) 51, 397-440.

Hertel, H., 1967. Biologisch-technische Forschungen über strömungstechnisch optimale Formen. Teil 1. - VDI Z. 109, 837-840.

Hessler, R. R., 1982. The structural morphology of walking mechanisms in eumalacostracan crustaceans. - Phil. Trans. R. Soc. (B) 296, 245-298.

Holst, E. von, 1935. Die Koordination der Bewegung bei den Arthropoden in Abhängigkeit von zentralen und peripheren Bedingungen. - Biol. Rev. 10, 234-261.

Hughes, D. A., 1970. Some factors affecting drift and upstream movements of Gammarus pulex. Ecology 51, 301-305.

Hurley, D. E., 1968. Transition from water to land in amphipod crustaceans. - Am. Zool. 8, 327-353.

Kaestner, A., 1967. Lehrbuch der speziellen Zoologie. Fischer, Stuttgart, 1, 2, 849-1242.

Kaschek, N., 1984. Vergleichende Untersuchungen über Verlauf und Energetik des Sprunges der Schnellkäfer (Elateridae, Coleoptera). - Zool. Jb. (Allg. Zool. Physiol. Tiere) 88, 361-385.

Kils, U., 1979. Schwimmverhalten, Schwimmleistung und Energiebilanz des antarktischen Krills, Euphausia superba. - Ber. Inst. Meeresk. Kiel 65, 1-79.

Kinzelbach, R, 1972. Zur Verbreitung und Okologie des Süßwasser-Strandflohs Orchestia cavimana Heller, 1865 (Crustacea: Amphipoda: Talitridae). - Bonn. zool. Beitr. 23, 267-282.

Kohlhage, K., 1983. Lokomotionsstudien an schwimmenden Copepoden. Dipl.-Arb., Münster, $87 \mathrm{pp}$.

Kühl, H., 1933. Die Fortbewegung der Schwimmkrabben mit Bezug auf die Plastizität des Nervensystems. - Z. vergl. Physiol. 19, 489-521.

Lawrence, R. F., 1953. The biology of the cryptic fauna of forests. Balkema, Cape Town, $408 \mathrm{pp}$.

MacMillan, D. L., Silvey, G. \& Wilson, I. S., 1981. Coordination of the movements of the appendages in the Tasmanian mountain shrimp Anaspides tasmaniae (Crustacea; Malacostraca; Syncarida). - Proc. R. Soc. (B) 212, 213-231.

Manton, S. M. 1977. The Arthropoda: Habits, functional morphology, and evolution. Clarendon, Oxford, $527 \mathrm{pp}$.

Meadows, P. S. \& Reid, A., 1966. The behaviour of Corophium volutator(Crustacea: Amphipoda). J. Zool. 150, 387-399.

Mortensen, T., 1921. Biologiske Studier over Sandstrandsfaunaen, saerlig ved de danske Kyster. Vidensk. Meddr dansk naturh. Foren. 74, 23-56.

Nachtigall, W., 1977. Zur Bedeutung der Reynoldszahl und der damit zusammenhängenden strömungsmechanischen Phänomene in der Schwimmphysiologie und Flugbiophysik. Fortschr. Zool. 24, 13-56.

Nursall, J. R., 1962. Swimming and the origin of paired appendages. - Am. Zool. 2, 127-141.

Pardi, L. \& Papi, F., 1961. Kinetic and tactic responses. In: The physiology of Crustacea. Ed. by T. H. Waterman. Acad. Press, New York, 2, 365-399.

Paul, D. H., 1971. Swimming behaviour of the sand crab, Emerita analoga (Crustacea. Anomura). I. Analysis of the uropod stroke. - Z . vergl. Physiol. 75, 233-258.

Paul, D. H., 1981. Homologies between body movements and muscular contractions in the locomotion of two decapods of different families. - J. exp. Biol. 94, 159-168. 
Remane, A., 1955. Morphologie als Homologienforschung. - Verh. dt. zool. Ges. 48, 159-183. (Zool. Anz., Suppl. 18.)

Remane, A., 1961. Gedanken zum Problem: Homologie und Analogie, Praeadaptation und Parallelität. - Zool. Anz. 166, 447-465.

Sars, G. O., 1895. An account of the Crustacea of Norway, Vol. 1: Amphipoda (Plates). Cammermeyer, Christiania, $248 \mathrm{pl}$.

Schellenberg, A., 1929. Körperbau und Grabweise einiger Amphipoden. - Zool. Anz. 85, 186-190.

Schellenberg, A., 1938. Brasilianische Amphipoden, mit biologischen Bemerkungen. - Zool. Jb. (Syst. Okol. Geogr. Tiere) 71, 203-218.

Schellenberg, A., 1942. Flohkrebse oder Amphipoda. 4. - Tierwelt Dtl. 40, 1-252.

Schlienz, W., 1922. Eine Süsswasser-Orchestia in der Aussenalster in Hamburg. - Arch. Hydrobiol. $14,144-150$.

Schrameck, J. E., 1970. Crayfish swimming: alternating motor output and giant fiber activity. Science, N.Y. $169,698-700$.

Segerstråle, S. G., 1946. On the occurrence of the amphipod, Gammarus duebeni Lillj. in Finland, with notes on the ecology of the species. - Commentat. biol. 9(18), 1-22.

Smallwood, M. E., 1903. The beach flea: Talorchestia longicornis. - Cold Spring Harb. Monogr. 1, $1-27$.

Smallwood, M. E., 1905. The salt-marsh amphipod: Orchestia palustris. - Cold Spring Harb. Monogr. 3, 1-31.

Spirito, C. P., 1972. An analysis of swimming behavior in the portunid crab Callinectes sapidus. Mar. Behav. Physiol. 1, 261-276.

Stephensen, K., 1938. Amphipoda, Tanaidacea und Pycnogonida. - Senckenbergiana 20, 236-264.

Verwey, J., 1927. Einiges aus der Biologie von Talitrus saltator (Mont.). - Int. Congr. Zool. 10, $1156-1162$.

Vester, H., 1986. 1900. Beiträge zur Kenntnis der Gattung Phronimopsis. Diss., Leipzig, 31 pp.

Vogel, F. Die abdominale Muskulatur von Orchestia cavimana Heller, 1865 (Crustacea, Amphipoda, Talitridae). - Crustaceana 50 (im Druck).

Webb, P. W., 1979. Mechanics of escape responses in crayfish (Orconectes virilis). - J. exp. Biol. 79, 245-263.

Weihs, D., 1977. Periodic jet propulsion of aquatic creatures. - Fortschr. Zool. 24, 171-175. 